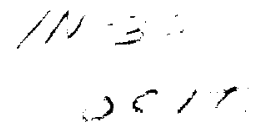

\title{
Flowfield Dynamics in Blunt Fin-Induced Shock Wave/Turbulent Boundary Layer Interactions
}

NASA Lewis Grant NAG3-1023

(March 13, 1989 - November 9, 1993)

\section{FINAL REPORT}

Prepared by:

\section{David S. Dolling \& Leon Brusniak}

January 1994

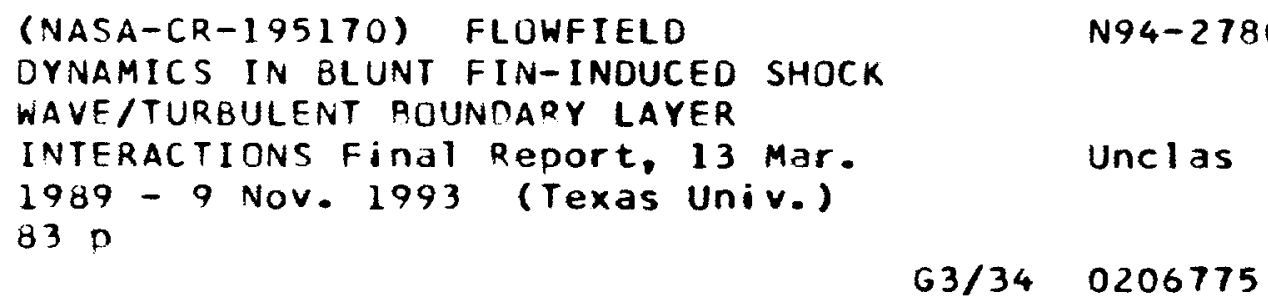

Center for Aeromechanics Research

The University of Texas at Austin Austin, Texas 78712 


\begin{abstract}
Fluctuating wall pressure measurements have been made on centerline upstream of a blunt fin in a Mach 5 turbulent boundary layer. By examining the ensemble averaged wall pressure distributions for different separation shock foot positions, it has been shown that local fluctuating wall pressure measurements are due to a distinct pressure distribution, $\mathcal{P}_{i}$, which undergoes a stretching and flattening effect as its upstream boundary translates aperiodically between the upstream influence and separation lines. The locations of the maxima and minima in the wall pressure standard deviation can be accurately predicted using this distribution, providing quantitative confirmation of the model. This model also explains the observed crosscorrelations and ensemble average measurements within the interaction. Using the $\mathcal{P}_{i}$ model, wall pressure signals from under the separated flow region were used to reproduce the position-time history of the separation shock foot. Further, the negative time delay peak in the cross-correlation between the predicted and actual shock foot histories suggests that the separated region fluctuations precede shock foot motion. The unsteady behavior of the primary horseshoe vortex and its relation to the unsteady separation shock are described.
\end{abstract}




\section{Contents}

$\begin{array}{ll}\text { Abstract } & 1\end{array}$

1 Introduction $\quad 2$

2 Experimental Program and Analysis Techniques 6

Wind Tunnel and Model . . . . . . . . . . . . . . . . . 6

Instrumentation and Data Acquisition . . . . . . . . . . . . 7

Test Procedure ................... 8

Statistical and Time Series Analysis ... . . . . . . . . . 10

Box-Car Transformation . . . . . . . . . . . . . . 10

Ensemble Averaging . . . . . . . . . . . . . . 11

Shock Foot History, $X_{s}(t) \ldots \ldots$. . . . . . . . . . . . . 12

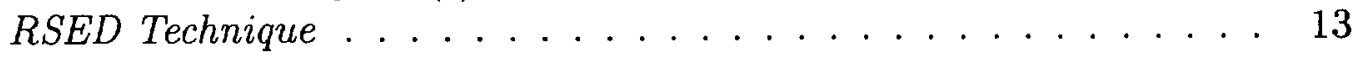

3 Discussion of Results 116

General Flowfield Features . . . . . . . . . . . . . . . . . . 16

Cross-Correlation Results: Analysis A . . . . . . . . . . . . . 17

Cross-Correlation Results: Analysis C . . . . . . . . . . . . . . . 20

Cross-Correlation Results: Analysis D . . . . . . . . . . . . . 21

Ensemble Average Results .................. . 23

Digital Filtering Results . . . . . . . . . . . . . . 25

4 Interpretation of Mean Pressure and Standard Deviation Distributions, Ensemble Averages, and Cross-Correlations 28

Global Flowfield Property $\left(\mathcal{P}_{i}\right)$ Hypothesis . . . . . . . . . . 28

Pressure Standard Deviation Estimation from $\mathcal{P}_{i} \ldots \ldots \ldots \ldots$

Ensemble Averages in Terms of $\mathcal{P}_{i} \ldots \ldots \ldots \ldots$. . . . . . . . . 31

Additional Ensemble Average Results . . . . . . . . . . . . 33

Cross-Correlations in Terms of $\mathcal{P}_{i} \ldots \ldots \ldots \ldots \ldots$

Analysis A Discussion . . . . . . . . . . . . . . . . 37

Analysis C Discussion . . . . . . . . . . . . . . . . . 39

Analysis D Discussion . . . . . . . . . . . . . . . . 40

Additional Remarks. . . . . . . . . . . . . . . . . . . 41 
5 Validation of Flowfield Model Through Prediction of Shock Foot Motion

6 Brief Comments on Practical Implications of Results 46

Computational Fluid Dynamics . . . . . . . . . . . . . . 46

Flow Control . . . . . . . . . . . . . . . . . . . 50

$\begin{array}{lll}7 & \text { Summary and Conclusions } & 51\end{array}$

8 Acknowledgements 53

9 References $\quad 54$

$\begin{array}{ll}\text { Figures } & 57\end{array}$ 


\section{Introduction}

Flow separation induced by shock wave/turbulent boundary layer interaction can pose significant problems in the design of high speed transport systems (Holden, 1986). One problem is the large fluctuating pressure loads, up to $185 \mathrm{~dB}$ or more (Dolling (1993)), associated with such interactions which can have characteristic frequencies close to the resonant frequencies of vehicle structural components (Pozefsky, et al., (1989)). Additionally, the high heating rates can further threaten the structural integrity of the vehicle. Consequential safeguards to overcome these local structural problems result in weight penalties which reduce the cost effectiveness and performance of the vehicle.

Shock-induced separation can arise from a variety of sources such as deflected elevons, engine inlets, wing-body junctures, and so on. In the laboratory these are typically modeled using geometries such as swept and unswept compression ramps, cylinders, and blunt fins. Some of the major features of these flowfields are illustrated in Fig 1 for the case of a hemicylindrically blunted fin of leading edge diameter D. In this example, there is a large scale, 3-D vortical, separated flowfield and, as is typically the case for shock-induced turbulent separation, it is highly unsteady. The unsteadiness is most visibly associated with the foot of the separation shock which undergoes aperiodic motion over a streamwise length of order $\mathrm{D}$, between the upstream influence line (UI) and the separation line 'S' (deduced using surface tracer flow visualization techniques) (Kussoy, et al., (1987), Dolling and Brusniak, (1991), Dolling and Bogdonoff, (1981,2), Dolling and Smith, (1989)). The result of this unsteady shock is clearly visible in the intermittent wall pressure signal of Fig 1 which is characterized by a low-amplitude, high frequency component associated with the 
undisturbed incoming turbulent boundary layer, a high-amplitude, low frequency component due to the passage of the shock foot over the transducer, and a high-amplitude, high frequency component associated with the flow downstream of the shock foot. The bandwidth of the shock foot-associated low frequency component is a few hundred $\mathrm{Hz}$ to several kHz (Dolling, (1993)).

Early experimental investigations of blunt fin-induced separation primarily provided mean wall pressure distributions and flow visualization images. Price and Stallings (1967) deduced the effects of fin leading edge sweep on the separated flow scale from mean wall pressure measurements and shadow photographs. Hussain (1985) similarly investigated the effects of sweep, in addition to angle of attack effects, and obtained highly resolved surface mean pressure distributions and surface flow visualization. As also seen in Price and Stallings' work, Hussain observed that the strongest effect of fin sweep occurred for sweep angles of 0 to $30 \mathrm{deg}$. and that "while bluntness intensified the interaction, sweep alleviated its intensity."

In the supersonic regime, Dolling and Bogdonoff $(1981,2)$ provided some of the first unsteady wall pressure measurements for unswept fins including standard deviation distributions and probability density distributions and determined that pressure fluctuation intensity increased with increasing $D / \delta$. However, single point measurements were done exclusively so that no correlations between various regions under the flowfield could be considered. Other, later studies have provided more detailed unsteady pressure measurement results (Narlo (1986), Dolling and Smith (1989), Dolling and Brusniak (1991), Gonsalez and Dolling (1993), Bamhart (1993), Kleifges and Dolling (1993)), including effects of leading edge sweep and sweep of the separation line ('S'), but only recently has the cause of the 
unsteadiness been specifically addressed. Dolling and Brusniak (1991) presented the results of simultaneous wall pressure measurements in the region of separation shock motion and under the separated flow in order to try and determine the relationship between fluctuations within the two regions. From this exploratory study, it was found that measurements from the region of shock motion correlated with measurements from the location in the separated flow at which the wall pressure standard deviation distribution attained a local maximum. In particular, the wall pressure signals were highly correlated between these two stations, and the energy spectrum at the separated flow station was very similar to the spectrum from the region of shock motion. In addition, it was found that shock foot motion correlated with certain pressure variations under the separated flow region and, in particular, that pressure "pulses" appeared to propagate upstream prior to changes of direction of the separation shock foot. However, a detailed physical explanation of these measurements and correlations was lacking.

Computational studies of unswept (Hung and Buning (1985)) and swept (Lakshmanan and Tiwari (1993)) blunt fin-induced separation have also been made. In the study of Hung and Buning, wall pressure distributions on and off centerline, and particle paths and pressure and Mach number contours in the plane of symmetry were calculated for a Mach 3 flow and compared with experimental data of Dolling, et al. (1979) and Dolling and Bogdonoff (1982). Overall, the comparisons were very good. In both of these computational studies the Reynolds-averaged Navier-Stokes equations, for which the random turbulence fluctuations are suppressed, were used. As such, direct or large-eddy turbulence simulation would be needed to resolve the oscillation (Hung and Buning (1985)). Because of this Hung and Buning concluded that "since the flow is sensitive to turbulence fluctuation and is inherently unsteady, the most important question is how the flow structure, 
such as horseshoe vortex and separation line, behaves in a stochastically oscillatory field." It has since been shown experimentally that the instantaneous separation location occurs just downstream of the separation shock foot and is essentially coincident with the shock foot as it moves, and that the separation line, 'S', obtained from surface tracer flow visualization indicates the downstream end of the region of shock motion (on centerline) (Gramann and Dolling (1988)); the horseshoe vortex behavior will be implied from this present study.

One of the practical goals of research in this area is to reduce the fluctuating pressure loads and high heating rates to levels that the vehicle structure can safely tolerate through the use of flow control devices and/or creative tailoring of vehicle component shapes. For computations to reproduce the effects of unsteadiness, the physics responsible for the unsteadiness needs to be ascertained so that they can possibly be included in the computational model. Experimentally however, before the physics can be determined, the physical origin of the fluctuating measurements themselves, be it from a horseshoe vortex or a subsonic separation bubble, must be understood: a simple cross-correlation result is meaningless unless it is understood why it has its characteristic shape. Understanding the origin of the fluctuating measurements (at a point) requires determining the global descriptive feature or features of the flowfield which cause the local measurements and their resulting properties. It is important to delineate between the fluctuating signal measured at a point from the continuous global, spatial, fluid flow phenomenon which is occurring, only a local segment of which is being monitored.

The fundamental objective of this research is to determine if a correlation exists between separation shock foot motion, pressure variations under the incoming undisturbed turbulent boundary layer, and pressure variations under the separated flow region, and to 
determine the source of the correlation. The specific tasks associated with this objective were: i) if such a correlation exists, explain the source of the correlation in terms of global flowfield properties; and ii) verify/clarify the global flowfield property model. Some implications of the results for flow control methods and flowfield computational methods are discussed. The results presented explain the findings presented in Dolling and Brusniak (1991) and provide insight into the effect of turbulence fluctuations on the horseshoe vortex as mentioned by Hung and Buning (1985).

\section{Experimental Program and Analysis Techniques}

\section{Wind Tunnel and Model}

The tests were conducted in the Mach 5 blowdown wind tunnel at the Wind Tunnel Laboratories of the University of Texas at Austin. The test section is 12 in. $(30.48 \mathrm{~cm})$ in length, 7 in. by 6 in. $\left(18 \mathrm{~cm}\right.$ by $15^{2} \mathrm{~cm}$ ) in cross-section, and is essentially a parallel wall extension connected to the end of the nozzle exit. The air is heated by two $420 \mathrm{~kW}$ banks of nichrome wire heaters upstream of the settling chamber. The floor of the test section was used as the test surface. Pressure transducers were mounted flush with the surface of a 3.375 in. $(8.5725 \mathrm{~cm})$ diameter rotatable plug. The plug had 26 transducer ports available on centerline at the minimum center-to-center spacing $\xi$ of 0.115 in. $(0.292 \mathrm{~cm})$ (Fig 1$)$. Measurements were taken upstream of the model on centerline.

The test model was a hemicylindrically blunted fin of 0.75 in. $(1.905 \mathrm{~cm}$ ) leading edge diameter. It had a $1.0 \mathrm{in} .(2.54 \mathrm{~cm})$ wide base extension which fitted into a matching slot in the floor with screws underneath holding the fin in place. This eliminated the need for 
a complicated sting support system which might have exacerbated tunnel blockage problems. The fin had a 0.5 in. $(1.27 \mathrm{~cm})$ streamwise range of travel. Gauge blocks in increments of $0.5 \xi$ were available for accurate and repeatable fin positioning relative to the transducer array. The overall fin height was 4.0 in. $(10.16 \mathrm{~cm})$. Based on the criterion of Dolling and Bogdonoff $(1981,1)$, at Mach 5 a height to diameter ratio of 6 is sufficient for a cylinder to be considered "semi-infinite."

The nominal freestream flow conditions and incoming turbulent boundary layer properties are shown in Table 1 . The floor surface temperature was within $8 \%$ of the adiabatic value. The boundary layer characteristics were determined by assuming a constant value of static pressure across it in conjunction with total temperature and pitot pressure surveys. A least-squares fit to the law of the wall/law of the wake following the procedure of Sun and Childs (1973) was used to obtain the velocity profiles. The turbulent boundary layer developed naturally without the use of trips.

\section{Instrumentation and Data Acquisition}

The fluctuating wall pressure signals were measured using Kulite miniature pressure transducers (models XCQ-062-15A and XCQ-062-50A) installed flush with the instrumentation plug surface. The Kulite transducers are absolute pressure sensors with a 0 to 15 psia and 0 to 50 psia range, respectively. They have a nominal outer case diameter of 0.064 in. $(0.1626 \mathrm{~cm})$ with a pressure sensitive silicon diaphragm 0.028 in. $(0.0711 \mathrm{~cm})$ in diameter. The diaphragm has a fully active four arm Wheatstone bridge diffused into it with a natural frequency of about $600 \mathrm{kHz}$ (as quoted by the manufacturer). The actual frequency response is limited to about $50 \mathrm{kHz}$ due to a perforated screen which protects the diaphragm 
from damage from dust and other particles. The transducer output was amplified and then electronically lowpass filtered at $50 \mathrm{kHz}$ before being digitized by LeCroy analog-to-digital (A/D) converters interfaced with a Hewlett-Packard 9000 series computer. The transducers were statically calibrated at least daily, and always after the transducers were repositioned.

The LeCroy data acquisition system has 2 12-bit A/D converters which operate on the same clock. Up to four channels per A/D can be sampled simultaneously at a maximum rate of $1 \mathrm{MHz}$ per channel, providing a maximum of 8 channels of simultaneously sampled data. Full scale A/D output is 0 to 4095 counts per channel. In the experiments, 8 channels of data were simultaneously sampled at either $200 \mathrm{kHz}$ or $500 \mathrm{kHz}$ with either 256,512 or 1024 records of data per channel being taken.

\section{Test Procedure}

Initially, flow visualization studies were conducted to determine the upstream influence and separation line locations so that the pressure transducers could be properly positioned. A variation of the kerosene-lampblack method was used. In this case, diesel fuel was added to reduce the volatility of the mixture. This ensured that the surface streak pattern did not set in its final form until well after steady freestream flow conditions were obtained.

The test runs involved simultaneous measurements under the different flowfield regions. Due to the eight channel limit, several runs were required to complete some of the five series of tests. Table 2 summarizes the test runs in terms of analysis type and transducer locations, where the spatial unit, $x$, is presented in multiples of $\xi$. As seen, transducers were located at various stations upstream of the fin root from $x=-0.5 \xi$ to $-26.5 \xi$ (refer also to Fig 
1). For analysis $A$ the reference transducer was held fixed under the incoming undisturbed flow at station $-26.5 \xi$ and the remaining seven transducers were positioned downstream of it. The objective was to relate the undisturbed flow measurements to those at successive downstream stations, through the interaction to the fin root, by examining the spatial relationship between fluctuations at the undisturbed flow station and at successive downstream stations. For analysis B the reference transducer was located at station $-16.5 \xi$, for which $\gamma \approx 0.9$ (intermittency, $\gamma$, is the fraction of the time that the separation shock foot is upstream of a given point). The objective of the series was to relate the intermittent region pressure fluctuations to the pressure fluctuations both upstream and downstream of it and to similarly see the successive spatial development between the intermittent station and the other two regions. Analysis $\mathrm{C}$ is essentially the same as analysis $\mathrm{B}$, except that the reference transducer was located at station $-18.5 \xi(\gamma \approx 0.5)$ in order to determine if the results depend on the reference transducer location. For the third series, analysis D, adjacent pairs of transducers at $1 \xi$ spacing were used to study the convection properties through the interaction. Finally, a single experiment was carried out to simultaneously monitor the majority of the intermittent region and a local portion under the separated flow at which the intermittent region and separated flow signals were known to be highly correlated. All runs were done at sampling frequencies of either $200 \mathrm{kHz}$ or $500 \mathrm{kHz}$; the number of records per channel ( 1 record $=1024$ data points) was either 256, 512, or 1024 (see Brusniak (1993) for complete details). Note that in analysis A, for the measurement at station $-0.5 \xi$, the upstream reference transducer was actually at station $-25.5 \xi$ since only 26 transducer ports were available; however, the results will only be affected by a slight timing difference since the undisturbed flow is still being measured exclusively (unlike the separated flow, for which measurements are a function of location). 
Statistical and Time Series Analysis

Statistical analysis consisted of calculations of the mean, standard deviation, the third and fourth order moments (i.e., skewness and flatness coefficients), and the amplitude probability density distribution of each channel, using the entire data array for a given channel. Time series analysis consisted of cross-correlations, auto correlations, coherence function, and power spectral density estimates (the definition and details of the calculation of these quantities can be found in Bendat and Piersol (1986)).

\section{Box-Car Transformation}

The wall pressure signals from the intermittent region are characterized by turbulence in the undisturbed and separated boundary layers, as well as a rise and fall in pressure when the shock crosses the transducer while moving upstream and downstream. The purpose of the conditional sampling algorithm (a threshold method) is to separate the shock wave component of the intermittent wall pressure signal from the superimposed turbulent components. The intermittent pressure signal is converted into a "box-car" form consisting of a series of 0's and 1's in which the 0's correspond to times when the shock wave is downstream of the transducer and 1's are when the shock is upstream of the transducer. The time at which the box-car changes from 0 to 1 is designated the rise time, $t_{r}$, since this is the time at which the separation shock foot crosses the pressure transducer while translating upstream, resulting in the pressure rise across the shock to be registered. A boxcar change in value from 1 to 0 is designated the fall time, $t_{f}$, which corresponds to the time at which the shock crosses the transducer while translating downstream, resulting in a fall in 
pressure. These times are indicated in Fig 1. The percentage of 1's in the box-car file is the intermittency, $\gamma$, of the signal at the given transducer location. The conditional sampling is not done in real time but on the digitized data stored on the computer. Early versions of the method were implemented by Narlo (1986) and later refined by Dolling and Brusniak (1989). The latter reference provides full details of the method, so only the basic ideas will be discussed.

The threshold algorithm begins by determining $\overline{\mathrm{P}}_{\text {wo }}$ and $\sigma_{\mathrm{Pwo}}$, the mean pressure and standard deviation of the undisturbed boundary layer component of the signal. Two thresholds are then defined: $\mathrm{T}_{1}=\overline{\mathrm{P}}_{\text {wo }}+(m) \sigma_{\mathrm{Pwo}}$, and $\mathrm{T}_{2}=\overline{\mathrm{P}}_{\text {wo }}+(n) \sigma_{\mathrm{Pwo}}$. From a sensitivity analysis in which both $m$ and $n$ were systematically varied, Dolling and Brusniak (1989) found that physically meaningful and reliable results are obtained for $m=3, n=6$ (i.e., $\mathrm{T}_{1}=\overline{\mathrm{P}}_{\text {wo }}+3 \sigma_{\mathrm{Pwo}}$, and $\mathrm{T}_{2}=\overline{\mathrm{P}}_{\text {wo }}+6 \sigma_{\mathrm{Pwo}}$ ). Using these threshold values the algorithm searches through the data file and determines the rise and fall times associated with each shock passage. This permits conversion of the signal to the box-car format. (Note. In application, only the rise and fall times are stored in the computer memory since a string of 0 's and 1's takes up considerable amounts of memory).

\section{Ensemble Averaging}

The purpose of ensemble averaging is to examine what correlation, if any, exists between separation shock motion and pressure variations under the incoming undisturbed turbulent boundary layer and under the separated flow region. Four specific shock motion "events" of interest were the uni-directional shock sweep (in both upstream and downstream directions) and shock changes of direction (downstream-to-upstream motion and upstream- 
to-downstream motion). At the lowest level, an upstream shock sweep occurs when the box-car of a given channel changes from 0 to 1 (time $t_{r}$ ), and vice versa for a downstream sweep (time $t_{\mathbf{f}}$ ). A downstream-to-upstream turn-around occurs when the time interval between a downstream sweep and the following upstream sweep occurs within a small time interval, $\Delta t$. An upstream-to-downstream turn-around is defined in a similar way.

The ensemble averaging analysis begins by taking pressure signals sampled simultaneously in the intermittent region and, for example, the separated flow region, searching for an "event" (such as an upstream sweep) on the intermittent (or trigger) channel and examining the signal on the downstream channel at the same time. A "time window" of variable width is set about the specific event of interest on the intermittent channel so that information not related to the event is excluded, providing a single ensemble for that channel. The time axes of both channels are then redefined with respect to the rise time, $t_{r}$, which is assigned value $\tau=0$ such that the original timing between channels is retained. The same window width from the intermittent channel is applied to the separated flow signal, providing the ensemble for that channel as well. The final step is to take all ensembles of each respective channel, "align" them about each respective $\tau=0$ reference, and average them to generate the ensemble averaged result for each channel.

Shock Foot History, $X_{s}(t)$

A detailed discussion on obtaining the separation shock foot position-time history, $X_{s}(t)$, from multi-channel pressure data can be found in Erengil and Dolling (1992) so only a brief description will be given here. 
$\mathrm{X}_{\mathrm{s}}(\mathrm{t})$ is obtained from multi-channel wall pressure measurements on centerline, where the transducers span the intermittent region at minimum center-to-center spacing $\xi$ (Fig 2a, compression ramp illustration, from Erengil and Dolling (1992)). The box-cars from each channel can be combined into a nested sequence as shown in Fig $2 b$ for the case of eight intermittent channels. Separate bins are established with boundaries extending from the downstream end of a given transducer to the downstream end of the adjacent transducer. For the present case there are six bins, with bin number 1 being the upstream-most bin, and the rest being numbered consecutively (Fig $2 \mathrm{c}$ ).

$X_{s}(t)$ is obtained from the nested boxcars by linear interpolation between successive rises or successive falls. In cases in which a rise is followed by a fall, which corresponds to an upstream-to-downstream change of direction, the $X_{s}(t)$ segment between the consecutive rise and fall is obtained by assuming that the shock foot moves at constant speed to the center of the next bin, changes direction, and then returns at constant speed to the original bin. The same process is applied to downstream-to-upstream changes of direction. Between successively detected rise or fall times there is no information about shock motion due to the discrete spatial sampling so that frequency decompositions of the $X_{s}(t)$ history must be viewed with caution due to this low-pass filtering effect. Velocity histories (Fig $2 \mathrm{~d}$ ) may be obtained by taking the distance between bins and dividing by the time between consecutive events (rises, falls).

RSED Technique

In this work events in the fluctuating pressure signals, such as from the incoming turbulent boundary layer, have been detected using a newly developed techniqued termed 
the Random Signal Event Detection (RSED) technique. It is a simple algebraic method which requires two streamwise separated, simultaneously sampled signals. Both arrays of data are subdivided into $\mathrm{N}$ contiguous segments of typically 100 to $200 \mu$ s duration. As an example, the undisturbed flow ensemble averages associated with upstream and downstream shock foot sweeps (discussed later) are characterized by sharp changes in pressure, where the signatures contain maximum (high point) and minimum (low point) values. In each successive array segment the time at which a maximum (or minimum) value occurs in the upstream-most channel is designated $\tau_{1}$, and the time at which a maximum (or minimum) value occurs in the other channel (downstream of the first) is designated $\tau_{2}$. The time difference $\tau_{2}-\tau_{1}$ from each parallel segment is added to a histogram array until all segments have been searched. For segments which are $100 \mu \mathrm{s}$ in duration, all $\tau_{2}-\tau_{1}$ values will fall between -100 and $+100 \mu \mathrm{s}$. The resulting histogram from two essentially undisturbed turbulent boundary layer signals is shown in Fig 3 for the maximum value case. In this example the transducers were spaced $3 \xi$ apart streamwise. The minimum value case is almost identical except that the maximum at $7 \Delta \mathrm{T}$ is about $28 \%$ smaller. As seen, the distribution is dominated by a large spike centered at $7 \Delta \mathrm{T}$, where $\Delta \mathrm{T}=2 \mu \mathrm{s}$ is the sampling time interval for this example, and the spike is defined by about five data points. The bounding values of the spike (where it basically rises above the surrounding "noise" level) can be understood by considering convection velocities. The maximum convection velocity for a turbulent structure is essentially $U_{\infty}$, the freestream velocity. For a $3 \xi$ spacing this gives a lower bound of $11 \mu \mathrm{s}(5.6 \Delta \mathrm{T})$ which is used to establish the reference level in the figure. The upper bound in the distribution, as determined from the reference level, is between 8 and $9 \Delta \mathrm{T}$, corresponding to convection velocities, $\mathrm{U}_{c}$, of $0.40 \mathrm{U}_{\infty}$ to $0.63 \mathrm{U}_{\infty}$ respectively, so that the events of interest (maximum or minimum values in the signals) convect at velocities in the range $0.40 U_{\infty} \leq U_{c} \leq U_{\infty}$. The most probable events take $14 \mu \mathrm{s}$ 
$(7 \Delta \mathrm{T})$, corresponding to $0.81 \mathrm{U}_{\infty}$. The standard convection velocity of $0.75 \mathrm{U}_{\infty}$ (deduced from cross-correlations) corresponds to $15 \mu \mathrm{s}(7.5 \Delta \mathrm{T})$ which falls between $7 \Delta \mathrm{T}$ and $8 \Delta \mathrm{T}$ in the histogram, so that the convection velocity is bracketed between these values.

As explained, the Fig. 3 histogram is physically reasonable. An implication of the result is that only those events in the boundary layer which have a lifespan greater than some minimum value will be detected. That is, the further apart the transducers are spaced, the lower the number of events contributing to the spike so that only the most coherent events will be detected (of more significant interest, these are the events which have a higher probability of remaining coherent up to when they reach the separation shock foot). This is analogous to the decay in the maximum cross-correlation coefficient with increasing streamwise spacing. Now that significant events in the flow (those traveling at $0.75 \mathrm{U}_{\infty}$ ) can be detected, a pseudo-boxcar can be created by assigning a value of 1 to the time at which the event (maximum) is located into the pseudo boxcar.

It can be argued that a maximum or minimum detected in given segment could possibly not be part of a sharp rise but instead part of a slowly varying signal which has a duration larger than the segment size. Nevertheless, the current method has been used since the results thus far are consistent with the flow physics and since the maximum or minimum detected in a slowly varying segment will likely appear in the noise portion of the histogram. 


\section{Discussion of Results}

\section{General Flowfield Features}

Fig 4a shows the mean pressure distribution on centerline upstream of the fin (the solid line is simply to aid in seeing the variations and is not a least-squares fit to the data). The spatial dimension, $\mathrm{x}$, is in multiples of $\xi$, the minimum center-to-center transducer spacing, since the wall pressure measurements were all taken at distinct multiples of $\xi$ as seen in Table 2. Physically, it would be most appropriate to normalize $\mathrm{x}$ by fin thickness, $\mathrm{D}$, but the spatial units would then be several digits in length, making it more cumbersome for following the discussion. However, for reference such a scale is shown underneath the $\xi$ scale.

The distribution is characterized by the initial increase above the undisturbed level at about $-23.5 \xi$ up to a maximum value near $-13.5 \xi$. The distribution is relatively flat from $-13.5 \xi$ to $-9.5 \xi$, then decreases rapidly at first (station $-8.5 \xi$ ), and then more gradually, until approximately $-3.5 \xi$. The pressure rises rapidly near $-3.5 \xi$; beyond this it is difficult to resolve the distribution due to this steep gradient. It is probable that the stations at $-1.5 \xi$ and $-0.5 \xi$ bracket a local maximum in the distribution, where the $-0.5 \xi$ station is on the decreasing portion. This is supported by the presence of a local maximum in earlier measurements of $\bar{P}_{w}(x)$ distributions (i.e., Fig 2 of Dolling and Bogdonoff $(1981,1)$ ).

The initial increase in $\overline{\mathrm{P}}_{\mathrm{w}}(\mathrm{x})$ is due to the increasing fraction of the wall pressure signal being dominated by the higher pressure levels downstream of the separation shock foot. The upstream-most extent of the shock foot motion near $-23.5 \xi$ is referred to as the 
upstream influence location (UI), and the downstream-most extent of the motion is indicated in Fig $4 \mathrm{a}$ by ' $\mathrm{S}$ ' at $-15.8 \xi$, the separation location obtained using surface tracer techniques (fluctuating wall pressure measurements bracket the downstream boundary between $-14.5 \xi$ and $-15.5 \xi)$. As described earlier, the intermittency, $\gamma$, at a point is the fraction of the time that the shock foot is upstream of the point and the flow is disturbed. The $\gamma=0.5$ location is indicated in the figure. As seen the data have some scatter. This is due largely to the difficulties of measuring mean pressures in the 0 to 2 psia range using transducers having a 0 to $15 \mathrm{psia}$ or 0 to $50 \mathrm{psia}$ range. The wide range is necessary to ensure high frequency response; lower range transducers have more flexible diaphragms with lower natural frequencies and lower usable frequency range.

Fig $4 \mathrm{~b}$ shows the standard deviation distribution. It is characterized by an increase beginning at UI, a maximum at $-17.5 \xi$, a decrease to a minimum at $-13.5 \xi$ and an increase to a plateau region which begins near $-10.5 \xi /-9.5 \xi$. As was seen in the $\overline{\mathrm{P}}_{w}(x)$ distribution, a rapid increase in $\sigma_{\mathrm{Pw}}(\mathrm{x})$ occurs also near $-3.5 \xi$, and similarly, a local maximum apparently exists between the $-1.5 \xi$ and $-0.5 \xi$ stations. In contrast with the $\overline{\mathrm{P}}_{w}(\mathrm{x})$ distribution, the $\sigma_{\mathrm{Pw}}(\mathrm{x})$ distribution has little scatter since the mean value has been subtracted from the data for the calculation.

Cross-Correlation Results: Analysis A

The cross-correlation results from analysis A are summarized in Fig 5. Recall that, for this case, the reference transducer is fixed under the undisturbed turbulent boundary layer at station $-26.5 \xi$ and simultaneous measurements are made at successive stations downstream of it. Seven characteristic cross-correlations are evident, with representative 
results being shown in the figure. (Note. The ranges over which each cross-correlation is representative are indicated in the figure; the complete set of figures is presented in Brusniak (1993)). The most obvious result is that a correlation does exist between the incoming undisturbed boundary layer flow and both the intermittent and separated flow regions. Each cross-correlation tends to have one, or both, of two modes: a broad mode due to low frequency components in the measured signals and a sharp mode due to high frequency contributions. Curve 1 has only a sharp mode whereas curve 2 has both, the broad mode having an overall negative $R_{x y}$. Curve 3 returns basically to a sharp mode. For the range of stations represented by curve 4 the sharp mode is always present, whereas the broad mode (with positive $\mathrm{R}_{\mathrm{xy}}$ ) is first discernible at about $-11.5 \xi$, is most prominent at $-9.5 \xi$, and decays to small levels again by $-4.5 \xi$. Beyond this station the features of the cross-correlations change rapidly. Curve $5(-3.5 \xi)$ has essentially a dual sharp mode with positive and negative $\mathrm{R}_{\mathrm{xy}}$, followed by curve $6(-2.5 \xi)$ which returns to a bimodal shape with negative $\mathrm{R}_{\mathrm{xy}}$ and finally curve $7(-0.5 \xi)$, which also is bimodal, but with a broad mode with positive $R_{x y}$. By defining a "transition" point as a location at which the character of the cross-correlation changes, it is evident that stations $-14.5 \xi /-11.5 \xi$ and $-3.5 \xi$ fit this definition, since the former separates regions of well-defined cross-correlations spanning approximately $6 \xi$ to $7 \xi$ in range with the latter at the downstream end of such a region. By regarding curve 6 as initiating a new region following transition point $-3.5 \xi$, then an additional transition point would apparently occur downstream of station $-1.5 \xi$, perhaps between curves 6 and 7 at about $-1.0 \xi$ or perhaps at station $-0.5 \xi$ (curve 7). These transition points are indicated in Table 3. The broad mode feature of the cross-correlation will be discussed in more depth later. The existence of a broad mode in the cross-correlation, especially as represented by curve 4 , suggests that a low frequency component must exist in the incoming undisturbed boundary layer signal. This feature will also be discussed later in the 'Digital Filtering 
Results' section.

It is evident from curves 1 through 5 that the sharp mode always has a positive value of $\mathrm{R}_{\mathrm{xy}}$ and positive time delay up to station $-3.5 \xi$. The data from station $-23.5 \xi$ (curve 1 ) is dominated by undisturbed boundary layer flow so that the sharp mode is due to the downstream convection of turbulent eddies in the boundary layer. The time delay of the maximum corresponds to a convection velocity of $0.75 \mathrm{U}_{\infty}$, which agrees with the typical broad band convection velocity for an undisturbed turbulent boundary layer. The fact that the sharp mode is present from stations $-22.5 \xi$ to $-3.5 \xi$ and that it occurs at progressively later time delay is evidence of the convection of eddies in the incoming boundary layer into the separated shear layer and through the interaction, at least to station $-3.5 \xi$. In that sense, the sharp mode can then be considered as a distinct perturbation on the broad mode portion of the cross-correlation so that the sharp and broad modes can be considered separately.

The results of time delay calculations using the RSED technique on the same data set are indicated in Fig 5 as either " $M$ " (maximum point criterion) or " $m$ " (minimum point criterion) and they agree well with the sharp mode peaks. In addition, Fig 6 shows the time delay as a function of location from both the cross-correlation and RSED results. The close agreement in the figure indicates that the RSED technique is capable of tracking events in the incoming undisturbed flow as they enter and pass through the interaction. One interesting feature is that after the structures have reached station $-10.5 \xi$, the maximum point results are always smaller in value than the minimum point results. This would seem to indicate an acoustical-type effect in which the maximum point values, which would generally be at a slightly higher pressure than the minimum point values, will also have a correspondingly higher temperature and hence, higher speed of sound. Because of this, the 
maximum point values travel at a slightly higher velocity.

\section{Cross-Correlation Results: Analysis C}

The results from analysis $\mathrm{C}$ are summarized in Fig 7. Recall that for this case, the reference transducer was located at station $-18.5 \xi$ (the middle of the intermittent region). Just as for analysis A, analysis C yields seven characteristic cross-correlations, demonstrating that a correlation does exist between the intermittent region and the incoming undisturbed flow (already seen in analysis A) and between the intermittent and the separated flow regions. The curves are primarily bimodal in character, having a broad and sharp mode. Curve 1 has a positive valued sharp mode and a broad negative mode; this curve is fundamentally the same as the result already shown as curve 2 in Fig 5 except that now the intermittent region station is the reference station, the timing is reversed. Curve 2 of Fig 7 is also bimodal, but now with a positive valued broad mode. Curve 3 has characteristics of both curve 2 and curve 4 , where curve 4 is of negative valued broad mode. The crosscorrelation character changes again between stations $-4.5 \xi$ and $-3.5 \xi$ (see curve 5) with only a broad mode apparent, and then changes again for stations $-2.5 \xi$ and $-1.5 \xi$ (curve 6) as a positive valued broad mode and changes once more at station $-0.5 \xi$ (curve 7 ) where it becomes a negative valued broad mode. Station $-14.5 \xi$ (curve 3 ) is clearly a transition point and station $-4.0 \xi$ (curve 6 ) is apparently another. Because of the change in character from station $-1.5 \xi$ to $-0.5 \xi$ (curves 6 to 7 ), station $-1.0 \xi$ is the approximate location of a third transition point. These stations are indicated in Table 3. The $\mathrm{R}_{x y}$ maximum value for the broad mode as a function of location is shown in Fig 8. As seen, the transition points correspond to the locations at which the cross-correlation coefficient changes sign and are as such related to the low frequency (i.e., broad mode) component in the data. 
The sharp mode is distinguishable up to station $-11.5 \xi$ as a distortion on the broad mode in the intermittent region results and apparently continues to be evident as a distortion on the broad mode up to approximately $-4.5 \xi$. The RSED results tend to agree with this (see arrows, curve 4). In Fig 5 the sharp mode (due to turbulent boundary layer convection) was clearly distinguishable for a larger distance since the reference station signal measures the undisturbed incoming flow exclusively so that the structures are more readily tracked. The convective feature is still evident from analysis C, especially for stations $-26.5 \xi$ to $-16.5 \xi$ where the sharp mode occurs as a distortion in the broad mode. It appears first for $\tau<0$ (e.g., station $-21.5 \xi$, curve 2 ) and then the distortion progressively appears at increasing values of $\tau$, appearing at time $\tau>0$ for stations downstream of the reference transducer. The sharp mode then is attributed to the convection of turbulent structures. The sharp mode is not always of positive value (see curve 4 ) as was the case in Fig 5. Since the sharp mode tends to have a weaker correlation then it would be significantly affected by the strong broad mode. Albeit, the timing of the sharp mode agrees with the RSED calculations. In light of these results the sharp mode is again seen to be a distinct perturbation on the broad mode. Analysis B produced essentially the same results as analysis C. The transition points are shown in Table 3.

\section{Cross-Correlation Results: Analysis D}

The results from the last cross-correlation analysis, analysis $D$, for which adjacent pairs of transducers at $1 \xi$ spacing were positioned throughout the centerline flowfield, are summarized in Fig 9. There are apparently only four basic types of cross-correlations (curves 1,2,5, and 7) but subtle features of the broad mode necessitate showing seven cases. Curve 1 is from undisturbed flow and has a single sharp mode associated with eddy 
convection. Curve 2 is dominated by a broad mode; a sharp mode is only weakly visible, in some cases as a perturbation, similar to what occurred in analysis C. The sharp mode becomes evident once more in curves 3 and 4 (strongest at station $-13.0 \xi$, curve 3 , weakest at station $-9.0 \xi$, curve 4 ). In curve 5 , from station $-4.0 \xi$, the broad mode has essentially disappeared. Curve 6 has a broad mode in addition to a sharp mode, and curve 7 has two sharp modes of positive and negative value. In curves 3 through 5 the sharp mode is seen to consist of a strong primary mode accompanied by smaller side lobes at positive and negative time delay. These side lobes are only weakly present in curve 2 and decay rapidly in curve 6 , being undetectable in curve 7 . In most of these cases the sharp mode again appears as a distinct perturbation on the broad mode.

The broad mode undergoes some obvious and some subtle changes through the interaction. It is dominant for stations $-22.0 \xi$ to $-16.0 \xi$, beyond which its width decreases in extent, attaining minimum width at station $-13.0 \xi$ (curve 3 ). Then, the width increases to a maximum value again at station $-9.0 \xi$ (curve 4 ), and then decreases once more in size and magnitude until it is almost indiscernible at station $-4.0 \xi$ (curve 5). It rapidly increases again in width and magnitude at station $-3.0 \xi$ (curve 6) and apparently becomes weakly negative for station $-1.0 \xi$ (curve 7). The rapid variations in cross-correlation shapes at the latter two stations classify them as being transition points. Because of the trends observed at stations $-13.0 \xi$ and $-9.0 \xi$ these two stations will also be designated as transition points. These points are listed in Table 3. It is seen now that the sharp mode is strong at station $-13.0 \xi$ and weak at station $-9.0 \xi$ because the broad mode is smallest and largest at the respective stations and causes either an accentuation or masking effect. 


\section{Ensemble Average Results}

The ensemble average results from analysis C, Fig 10, show seven characteristic pressure signatures associated with upstream and downstream shock foot sweeps. The solid horizontal lines in the figure are the mean pressure levels of the entire signal at the given stations and will be referred to later. For the upstream sweep case, the incoming undisturbed flow result is characterized by an "S" shaped signature of small amplitude and short duration, as seen in curve 1(u) just to the left of $\tau=0$. Ensemble averages from the intermittent region (curve 2(u)) have a low level corresponding to undisturbed flow followed by a rapid rise to higher levels due to passage of the shock foot over the pressure transducer. Although this broad signature dwarfs the undisturbed flow signature, the latter is still evident (around $\tau=$ 0 ). The character of the ensemble averages changes rapidly at station $-14.5 \xi$ to the peaked signature shown in curve $3(\mathrm{u})$. The peaked character is strongest at this station; proceeding downstream the rising portion to the immediate left of the peak increases in level such that the peak becomes no longer discernible by about station $-11.5 \xi$. From here to station $-4.5 \xi$ the ensemble averages are characterized by a broad drop in pressure levels (curve $4(u)$ ). Station $-3.5 \xi$ (curve $5(\mathrm{u})$ ), which is characterized by a low level and then a rise in pressure, is apparently the beginning of a change in character from falling levels (curve $4(\mathrm{u})$ ) to the broad rising pressure characteristic of the station immediately downstream of it (station $-2.5 \xi$, curve $6(\mathrm{u}))$. There is a change in character once more between stations $-1.5 \xi$ and $-0.5 \xi$ to one of a broad falling pressure (curve $7(u)$ ). This change likely occurs between these two stations since no "combination" of trends is evident. As such, stations $-1.0 \xi$ and $-4.0 \xi$ are designated as transition points. Station $-14.5 \xi$ is also so designated since it is the station at which its peak is first clear. These points are indicated in Table 3. 
The sharp signature, 'S', seen in curve $1(\mathrm{u})$ is also evident in curve $2(\mathrm{u})$, but, as seen, is dwarfed by the large pressure increases across the shock foot. Its passage through the interaction is still evident in curves $3(u)$ and $4(u)$ as a small perturbation just to the right of $\tau$ $=0$ but is difficult to discern due to the presence of other similar perturbations in the ensemble averages. The RSED results indicate where the peaks corresponding to boundary layer convective phenomenon should occur. As such, they help in discerning which of the sharp variations in the ensemble averages are associated with boundary layer convection and which are not.

For the downstream sweep case the above discussion holds analogously. The primary difference is that the results, for the most part, are mirror-images about $\tau=0$ of the upstream sweep results: The curve 1(d) sharp signature is now a "backward $S$ " shape, the curve 2(d) signature is a broad fall in pressure as the shock foot crosses to downstream of the pressure transducer; in curve 3(d) the pressure levels to the left of the peak now fall and instead the pressure levels to the right of the peak increase in level; the broad changes in curves 4(d), $6(d)$, and $7(d)$ are now opposite in nature to the upstream sweep case; curve 5 (d) is apparently just downstream of a transition point.

One curious observation is the following: for the case shown in Fig 10, the upstream and downstream sweep ensemble averages all correspond to the same event triggering location. That is, the ensemble averaging is always done when the shock foot is at the same station, irrespective of direction of motion. With this in mind, then, why are curves $3(u)$ and 3(d), for example, not the same (the peaks occur on opposite sides of $\tau=0$ )? In particular, if the shock foot is at the same location when ensemble averaging is done, why are the ensemble average results different? This question will be re-addressed later in the 
'Additional Ensemble Average Results' discussion of section 4.

The analysis B results are essentially the same as for analysis $\mathrm{C}$, and the transition points are included in Table 3.

\section{Digital Filtering Results}

Earlier, in the 'Cross-Correlation Results: Analysis A' section, the issue of a low frequency component existing in the incoming undisturbed boundary layer signal arose. In this section this issue is addressed by lowpass and highpass filtering of the digital data using an FIR non-recursive filter. In the analysis (presented in Brusniak (1993)), filter cutoff frequencies of 4,6,10,20 and $30 \mathrm{kHz}$ were used and were based upon the shape of the power spectrum at the $-10.5 \xi$ station. The highest value used was $30 \mathrm{kHz}$ since the analog filters used during data acquisition were set at a lowpass cutoff of $50 \mathrm{kHz}$.

As discussed in the analysis A section, the broad mode at station $-9.5 \xi$ (Fig 5, curve 4) implies the existence of a low frequency component in the undisturbed flow signal. The idea behind digital filtering is that, by applying a highpass filter to the undisturbed flow

signal, only frequencies in that signal greater than the digital filter cutoff frequency, $f_{c}$, would be retained and, for an appropriate cutoff value, the broad mode in the the crosscorrelation would be suppressed. As a complement to this highpass filter analysis, a lowpass analysis was also performed (only frequencies less than the digital filter cutoff would be retained) with the expectation that the sharp mode would eventually be suppressed.

The unfiltered cross-correlation from station $-10.5 \xi$ (almost identical to the station 
$-9.5 \xi$ result) is shown in Fig 11a, and the result from the highpass analysis is shown in Fig 11b. In the analysis $f_{c}$ was set first at the smallest value $(4 \mathrm{kHz})$ and sequentially increased in order to retain most of the whole range of frequencies in the signal at first, and to see the gradual change in the cross-correlation from the unfiltered case. At the first setting, $f_{c}=4$ $\mathrm{kHz}$, an abrupt removal of the broad mode from the cross-correlation occurred (Fig 11b). This result remained basically unchanged for values of $f_{c}$ up to and including $10 \mathrm{kHz}$, beyond which the magnitude of the cross-correlation was suppressed. In the lowpass analysis case, for which $f_{c}$ was first set to the highest value (again, to see the gradual effect), the effect on the sharp mode was gradual. As $f_{c}$ was successively set to lower values the sharp mode became increasingly suppressed until at $4 \mathrm{kHz}$ (Fig 11c) it is evident only as a minor distortion from around $\tau=0$ to $0.2 \mathrm{~ms}$ in the cross-correlation.

This digital filtering analysis was repeated but now for the ensemble average results (i.e., the same $\mathrm{f}_{\mathrm{c}}$ values were used) and was done by filtering the entire undisturbed flow data string before ensemble averaging. The goal of this analysis was to determine if a low frequency (broad signature) exists which correlates with separation shock motion.

The lowpass filter result is shown in Fig 12. As was done in the preceding crosscorrelation analysis, $f_{c}$ values were set first to the highest value and sequentially decreased. The effect was that the sharp signature ' $S$ ' in the upstream sweep ensemble average (solid line, Fig 12a) was gradually suppressed and then suddenly disappeared completely for $f_{c}=$ $10 \mathrm{kHz}$, leaving an ensemble average characterized by a broad dip in pressure values (solid line, Fig 12b) at just around $\tau=-0.1 \mathrm{~ms}$. The dip is small, but certainly physical, since its shape reverses for a change in shock direction of motion (see Fig 12e discussion, below). This low frequency signature is suppressed in magnitude with further decreases in $f_{c}$, but 
remains clear. For the downstream sweep case, just as for the upstream sweep case, as $f_{c}$ was increased the sharp 'backward S' signature (Fig 12d) was increasingly suppressed. In this case, at $f_{c}=10 \mathrm{kHz}$, the ensemble average is characterized by a broad hump in pressure values. This low frequency signature also becomes increasingly suppressed for further decreases in $f_{c}$, but from the sequence shown in the figure it is still evident. There is not as obvious an abrupt change in character when $f_{c}$ is decreased from 20 to $10 \mathrm{kHz}$ for the downstream sweep case as occurred in the upstream sweep case. This occurs since the low frequency hump (downstream sweep case, Fig 12e) is similar in shape to the unfiltered case (a "sharp hump", Fig 12d), whereas for the upstream sweep, it is easy to contrast the unfiltered sharp signature (Fig 12a) with the resultant low frequency signature dip of Fig $12 b$ (i.e., opposite characters). For $\mathrm{f}_{\mathrm{c}}=4 \mathrm{kHz}$, there is a gradual decreasing level for the upstream sweep case and, more so, of a gradual increasing level for the downstream sweep case from -0.4 to $0.4 \mathrm{~ms}$ (Figs $12 \mathrm{c}, 12 \mathrm{f}$ ). A similar small effect was observed in the compression ramp experiments of McClure (1992) where the undisturbed flow measurements were made in the flow using a fluctuating pressure pitot probe. It was observed that a gradual decrease in pitot pressure occurred during shock upstream sweeps and that an increase in pressure occurred during downstream sweeps. The total change (increase or decrease) was typically $25 \%$ of the fluctuating pitot pressure standard deviation and spanned at least $1 \mathrm{~ms}$ in time. For the present results the changes in pressure are less than roughly $8 \%$ of the wall pressure standard deviation value, but they also span at least 1 $\mathrm{ms}$ in time.

In summary, a low frequency component in the undisturbed flow signal is responsible for the broad mode of the station $-9.5 \xi$ cross-correlation of analysis A (curve 4, Fig 5). This component is of frequency or frequencies less than $4 \mathrm{kHz}$. From the ensemble average 
results it is seen that low frequency (broad) pressure signatures exist which correlate with shock motion and are a function of shock direction of motion.

\section{Interpretation of Mean Pressure and Standard Deviation Distributions, Ensemble Averages, and Cross-Correlations}

As was discussed in the Introduction, it is necessary to understand the global flowfield behavior in order to explain local (point) measurements and the behavior of the two-point correlations and ensemble averages. A global flowfield representation which attempts to synthesize the mean pressure and standard deviation distributions, and is able to show that the ensemble averages and cross-correlations are the result of a single global effect will be presented in this section.

Global Flowfield Property $\left(P_{i}\right)$ Hypothesis

The mean wall pressure distribution in Fig 4a gives a zeroth-order global description of the centerline pressure field. It is characterized by a rise to a local maximum value followed by a gradual decrease and then a rapid rise in value near the fin root. This description, however, does not account for the unsteady aspect of the flowfield. A global description which provides a first order inclusion of the unsteadiness is the ensemble average pressure distribution at time $\tau=0$. This time corresponds to the separation shock foot being located directly over the given intermittent region pressure transducer and therefore provides the ensemble averaged pressure distribution for the shock foot "fixed" at a given station; this distribution can be obtained for shock motion in the upstream and downstream directions. 
The ensemble averaged wall pressure distribution $\left(\overline{\mathrm{P}}_{\mathrm{EA}}(\mathrm{x})\right)$ for the upstream sweep case with the shock foot fixed at the downstream end of the intermittent region $(\gamma=0.9$, station $-16.5 \xi$ ) is shown in Fig 13. Both sweep cases provide basically the same result (for clarity only the upstream sweep result is shown) and essentially resemble the mean pressure distribution of Fig 4a, except that the initial pressure rise begins at the shock foot location, station $-16.5 \xi$. Data scatter is likewise a problem here, as in Fig 4a. The ensemble average wall pressure distribution for the shock foot fixed at an intermittency of 0.5 (station $-18.5 \xi$ ) is also shown in the figure and is also similar in shape to the mean wall pressure distribution. The difference is that the 0.5 intermittency distribution has been "stretched" further and the pressure levels are lower ("flatter").

Fig 13 illustrates that, although a distortion of the $\overline{\mathrm{P}}_{\mathrm{EA}}(\mathrm{x})$ distributions occurs for the two shock foot fixed cases, the distributions are essentially similar and resemble the mean wall pressure distribution. These results lead to the hypothesis that the local wall pressure (point) measurements and correlations are due to the effects of the global property of an "instantaneous" wall pressure distribution, $P_{i}$, corresponding to any given $\overline{\mathrm{P}}_{\mathrm{EA}}(\mathrm{x})$ distribution, the upstream end of which translates aperiodically and undergoes a stretching and flattening effect which is a function of where the shock foot is located in the intermittent region. Therefore, local pressure variations measured at a given point will be due largely to the range of pressures associated with a local segment of the $P_{i}$ distribution which likewise translates aperiodically over the measurement point.

Pressure Standard Deviation Estimation From $P_{i}$

If the $P_{i}$ model is correct, then it should be possible to predict properties such as the 
wall pressure standard deviation distribution. Consider a specific point on centerline downstream of the shock foot: as the shock foot translates back and forth, locally the wall pressure will increase and decrease, the magnitude depending on the local segment of $P_{i}$. Now, $\sigma_{\mathrm{Pw}}(\mathrm{x})$ is essentially a measure of the square root of the sum of the squares of the difference between each data point and the mean value; for a single data point it is basically the absolute value of the difference. In light of this, a measure of $\sigma_{\mathrm{Pw}}(\mathrm{x})$ can be obtained by calculating the absolute value of the pressure change at each station, $\mid \Delta \mathrm{PI}$, which occurs when the shock foot (the leading edge of $P_{i}$ ) changes from one end of the intermittent region to the other. The result of changing from a $\gamma$ value of 0.9 to 0.5 (or 0.5 to 0.9 ) is shown in Fig 14, where the solid line has been sketched as an aid to the eye. As seen, maximum values occur at about stations $-16.5 \xi,-9.5 \xi$ and near $-1.0 \xi$, and minimum values occur at about station $-13.5 \xi$ and near $-6.5 \xi$, in close agreement with the maxima and minima in the original $\sigma_{\mathrm{Pw}}(\mathrm{x})$ distribution which is also shown in the figure. As in Fig $4 \mathrm{~b}$, little scatter is seen in the $\mid \Delta \mathrm{PI}$ data. If additional runs had been available with the reference transducer at a station at which $\gamma$ is small, then the $|\Delta \mathrm{P}|$ distribution would fill out overall.

As seen, the $|\Delta \mathrm{P}|$ distribution overpredicted the local $\sigma_{\mathrm{Pw}}(\mathrm{x})$ measurements from about $-17.5 \xi$ to $-14.5 \xi$. The reason for this is that the shock foot by definition spends $50 \%$ of the time upstream (or downstream) of the $\gamma=0.5$ station, whereas it only spends $10 \%$ of the time downstream of the $\gamma=0.9$ station; consequently, the calculated $|\Delta \mathrm{P}|$ values will only appear for $10 \%$ of the time in the $-17.5 \xi$ to $-14.5 \xi$ station signals so that the $|\Delta \mathrm{P}|$ values are actually being overestimated (an alternative would be to use $P_{i}$ distributions from $\gamma$ values which encompass most of the shock foot motion, such as for $\gamma$ values of $0.2,0.5$ and 0.8 ). The underprediction for stations $-13.5 \xi$ to $-1.5 \xi$ is likely due to a decreasing correlation between the upstream end of $P_{i}$ and the downstream part of $P_{i}$ (i.e., disturbances associated 
with shock foot motion will be strongest near the shock, but weaker further away from the shock). If a series of $P_{i}$ distributions spanning ' $S$ ' to UI were available, then the shock foot intermittency distribution could be used in order to increase the accuracy of the $\sigma_{\mathrm{Pw}}(\mathrm{x})$ estimation. This simple result provides the first evidence in support of the $P_{i}$ model.

\section{Ensemble Averages in terms of $P_{i}$}

Table 4 summarizes the ensemble average and cross-correlation results using miniatures from Figs 5, 7, 9, 10,11 and 12. Each row, labeled (a) through (e) corresponds to the specified analysis set. Since there are basically seven distinct curves from each analysis set, they are indicated by the "curve numbers" at the top of Table 4. For this discussion only the ensemble average results in rows (a) and (b) will be addressed. (Note. The upstream and downstream sweep cases are distinguished by either a "(u)" or "(d)", respectively, affixed to the end of the curve number. Also, the curve 1 results in rows (a) and (b) show the three characteristic pressure signatures found from the digital filtering analysis results of Fig 12 , and are distinguished as being of character $\mathrm{i}$, ii, or iii, with the appropriate "(u)" or "(d)" notation).

From the $P_{i}$ model, the ensemble average sweep signatures should correspond to motion of local segments of $P_{i}$. Consider the upstream sweep case first (Table 4, row (a)). As seen in the curve $2(u)$ ensemble average, as the shock foot moves upstream over a given point the pressure increases from its undisturbed value. Now, when the front end of $P_{i}$ translates upstream, the pressure at a point on the surface just ahead of the upstream edge of $P_{i}$ will initially see an undisturbed level, followed by an increase, just as in the ensemble average result. Next, ensemble average curve $3(\mathrm{u})$ has a peak which is characteristically 
evident in the region $-14.5 \xi$ to $-11.5 \xi$. For a point in this region, as $P_{i}$ moves upstream, the local maximum in $P_{i}$ will cause the pressure at the point to increase, then decrease, just as in the ensemble average. The peak is less pronounced in this figure than for the analysis B results (not shown) due probably to the stretching and flattening effect. By continuing this analysis approach, it is clear that $P_{i}$ will produce decreasing pressure levels in curve 4(u), increasing levels as in curve 6(u), and decreasing levels as in curve 7(u). These last two features again imply that a secondary maximum in $\overline{\mathrm{P}}_{\mathrm{w}}$ (x) exists near the fin root, but the most striking evidence of this is the fact that, in addition to the overall broad increase in pressure in curve 6(u), a local peak in curve $6(\mathrm{u})$ which would be indicative of this local maximum is clearly visible, analogous to the peak in curve 3(u). (Note also from the $\overline{\mathrm{P}}_{\mathrm{EA}}(\mathrm{x})$ distributions (Fig 13, inset) that as the shock foot position is changed from the 0.9 intermittency to the 0.5 intermittency location, the pressure value at station $-1.5 \xi$ increases and the pressure value at station $-0.5 \xi$ decreases. This is entirely consistent with the prediction that a local maximum exists in the $\overline{\mathrm{P}}_{\mathrm{w}}(\mathrm{x})$ distribution between these stations). In addition, the curve $6(\mathrm{u})$ results suggests that this local peak is part of an overall increasing level (see $P_{i}$ sketch at top of Fig 16). The transition point figure, curve 5(u), is of small magnitude and has the beginnings of characteristics of curve $6(u)$ and is indicative of the passage of a local minimum in $P_{i}$.

For the downstream sweep case the above discussion holds analogously. In particular, for a point between stations $-21.5 \xi$ and $-15.5 \xi$, as $P_{i}$ shifts downstream a decreasing pressure level leveling off at the undisturbed level will be recorded, just as seen in the ensemble average result, curve 2(d) of row (b). The relationship between $P_{i}$ and the remaining downstream sweep curves agree as well. Curve 5(d), which is of small magnitude, does not quite show a minimum in $P_{i}$, but this is likely due to weak competing influences of the curve 4 and curve 6 pressure variations. However, its small magnitude of 
variation, compared to the magnitude of the pressure decrease at the $-2.5 \xi$ station just downstream of it, agrees with its being a local minimum.

By considering the Dolling and Brusniak (1991) ensemble averages "pulses" in light of these results it is clear that the source of the pulses are the result of a $P_{i}$ distribution. For a downstream-to-upstream shock foot motion the peak in the separated region ensemble averages occurs since, as the shock foot moves downstream the separated region transducers detect the falling portion of $P_{i}$ (downstream of its peak) so that the measured pressure increases with time; then; as the shock foot changes direction (to upstream), the measured pressure decreases, giving the peak "pulse" result. The minimum occurs for upstream-todownstream changes of direction since, as the shock foot moves upstream, the separated region transducers measure falling levels, followed by increasing levels as the shock foot changes direction of motion. The "pulses" therefore are not convective phenomena. The upstream "pulse" convection suggested by Dolling and Brusniak (1991) can now be interpreted correctly to mean that pressures induced by the vortex motion (and the resultant changes in measured pressure) precede changes in direction of the separation shock foot (i.e., that the separated flow is responsible for the shock foot unsteadiness).

\section{Additional Ensemble Average Results}

Before addressing the cross-correlation results, it is helpful to clarify the relationship between the ensemble average signatures and the physical character of the source of these signatures. As was just described, the ensemble averages from downstream of the shock foot can be related to $P_{i}$. For the undisturbed flow region upstream of the shock foot no single global model analogous to $P_{i}$ is available for explaining the character of the undisturbed flow 
ensemble averages. However, these signatures can at least be documented as local, distinct segments which convect past a given point to give curves 1, rows (a) and (b), of Table 4.

For the case of shock upstream sweep (row (a), curve 1-i(u) of Table 4 ) as $\tau$ (time) increases in the positive direction the wall pressure signature first decreases, then increases sharply, and finally drops to the original mean level. The type of convecting pressure signature which would give this type of pressure-time history would be a mirror image of this ensemble average result, just as the downstream sweep $P_{i}$ result is the mirror image of the upstream sweep result. The reason for this is that the time axis on the ensemble averages is always directed from left to right, however, $P_{i}$ can translate either upstream or downstream, giving opposite results (only the upstream motion results resemble the pressure-time histories). Similarly, then, since the undisturbed flow ensemble averages are the result of pressure signatures convecting in the downstream direction only, the results will be essentially similar to the $P_{i}$ downstream sweep motion case. In light of this discussion, Fig 15 shows the physical characteristics of the signature segments convecting into the interaction which would give the ensemble average results shown in Fig 12. That is, for a pressure transducer located downstream of the "convecting signature shape" in Fig 15, as the signature convects past the transducer it will cause the pressure variation shown in the ensemble averages.

One important issue is the relation between shock motion and pressure variations upstream and downstream of the shock foot. In Dolling and Smith (1989) the inviscid shock velocity magnitude $(\mathrm{W})$ relation to the pressure ratio $\left(\mathrm{P}_{2} / \mathrm{P}_{1}\right)$ across the oblique shock wave was presented to estimate fluctuations in $\mathrm{W}$ due to the fluctuations in pressure $\left(\sigma_{\mathrm{PW}}\right)$ both upstream and downstream of the separation shock foot. From this relation, where $\mathrm{W}=$ 
$f\left(P_{2} / P_{1}\right)$, as $P_{2} / P_{1}$ increases, $W$ increases and the shock moves upstream (since $\left(W-U_{\infty}\right)>$ 0 ); as $\mathrm{P}_{2} / \mathrm{P}_{1}$ decreases, $\mathrm{W}$ decreases and the shock moves downstream (since $\left(\mathrm{W}-\mathrm{U}_{\infty}\right)<0$ ). First consider the effects of $\mathrm{P}_{1}$ on shock motion (i.e., assume $\mathrm{P}_{2}$ to be constant). If the incoming undisturbed flow region is going to cause the shock foot to move upstream, then the value $P_{1}$ ahead of the shock must decrease with time; it must increase with time for downstream motion to occur. From Fig 15 it is clear that curves 1-ii(u) and 1-iii(u) of the upstream sweep ensemble average case (row (a) of Table 4) would be conducive to causing upstream motion of the separation shock. From the downstream sweep ensemble averages, curves 1-ii(d) and 1-iii(d) (row (b), Table 4) are conducive to downstream shock motion.

Because of the small magnitude of the fluctuations in the incoming undisturbed flow, curves 1 -i(u), 1-i(d), 1-ii(u) and 1-ii(d) of Table 4 would undoubtedly produce small changes in W $(\Delta W)$ and appear as a "jitter-type" motion as discussed by Erengil and Dolling (1992). However, since displacements in shock foot position are the time integral of the velocity difference of $\mathrm{W}$ and $\mathrm{U}_{\infty}\left(\Delta \mathrm{x}=\int\left(\mathrm{W}-\mathrm{U}_{\infty}\right) \mathrm{dt}\right)$, then small differences integrated over a long time period (as suggested by the long duration change in pressure in curves 1-iii(u) and 1iii(d)) could possibly be manifested as low frequency motion of the separation shock foot.

In the 'Ensemble Average Results' discussion section, the question as to why the peaks in the ensemble averages of curves 3(u) and 3(d) of Fig 10 occur on opposite sides of $\tau$ $=0$, can now be answered. In the upstream sweep case, the maximum occurs at time $\tau<0$. This means that, as the shock foot translates in the upstream direction, the maximum in $P_{i}$ crosses the separated region transducer before the shock foot crosses the event trigger transducer in the intermittent region. Consequently, at time $\tau=0$ the separated region transducer is sensing the downstream end of the $P_{i}$ maximum (see Fig 16). Next, for the 
downstream sweep case, when the shock foot is initially moving in the downstream direction (i.e., time $\tau<0$ ), the $P_{i}$ maximum is already upstream of the separated region transducer. Thus, when the shock just crosses over the intermittent region trigger transducer, time $\tau=0$, the separated region transducer is again measuring the downstream end of the $P_{i}$ maximum. Only after the shock foot has passed downstream of the trigger transducer (i.e., time $\tau>0$ ) is the presence of the $P_{i}$ maximum detected at the separated region station. Consequently, the $P_{i}$ peak occurs at time $\tau>0$ (Fig 16 ).

Cross-Correlations in terms of $P_{i}$

Before considering the detailed cross-correlation results it will be helpful to discuss some qualitative features of cross-correlation calculations. First, several model signals are given in Fig 17. Included are signals characterized by rising levels $(R)$, falling levels $(F)$, a rising-falling or peak $(\mathrm{P})$ signal, and a falling-rising or trough $(\mathrm{T})$ signal. When standard cross-correlations are calculated they are for zero-mean signals (or ones for which the mean level has been subtracted); the model signals account for this so that levels above the mean have a positive ("+") value and levels below the mean have a negative ("-") value, as shown in the figure. For the model signals the maximum cross-correlation value occurs for zero time delay. As seen, when the maximum cross-correlation value is calculated for the riserise (R-R) combination, the integral will be of an integrand which is always positive (for $\tau$ not equal to 0 ) since, for time $\tau<0$ both signals are negative and for $\tau>0$ both signals are positive; the cross-correlation maximum will be of positive value, as suggested in the figure. The same result is obtained for F-F, P-P and T-T combinations. Along similar lines, it is clear that R-F, F-R, P-T and T-P combinations will result in a negative maximum crosscorrelation value. Of course, the overall shape of the entire cross-correlation for any of the 
above model combinations can differ, but here the maximum value is what is of interest. With these qualitative cross-correlation relationships in mind, the detailed cross-correlation results can now be related to the ensemble average characteristic signatures, which in turn stem from $P_{i}$

\section{Analysis A Discussion}

The analysis A cross-correlation results are summarized in row (c) of Table 4. Consider first the non-transition point regions from the ensemble average results (rows (a) and (b), curves 1, 2, 4, 6 and 7). As seen, the ensemble averages are generally of a broad signature type with superimposed sharp mode variations; the broad mode results will be addressed first. Cross-correlation curve 2 (row (c)), between the incoming undisturbed flow and an intermittent region station, is characterized by a broad mode of overall negative value. From the qualitative models this would suggest a F-R or a R-F relationship between the two regions. From the curve 2(u) upstream sweep ensemble average results the intermittent region is characterized by a R-type signature and the incoming flow signature 1-iii(u) is apparently an F-type signature, in agreement with the qualitative expected relationship (F-R). For downstream sweeps the F-type behavior in the intermittent region and the R-type behavior in the incoming flow likewise agree (R-F). The fact that the relationship suggested by the cross-correlation can be seen in the ensemble averages adds validity to the existence of the subtle broad mode measured at the surface under the incoming undisturbed flow (curves 1-iii(u) and 1-iii(d) of rows (a) and (b)). Next, crosscorrelation curve 4 has a broad mode of overall positive value. This suggests that likebehavior occurs in both signals ( $R-R$ and/or F-F). This is indeed the case: both the incoming and separated region upstream sweep ensemble average signatures (curves 1 and 4) are of 
F-type; both are of R-type for the downstream sweep case. Continuing this approach, it is seen that the F-R (upstream sweep)and R-F (downstream sweep) combinations lead to the overall negative character in cross-correlation curve 6 and that the F-F (upstream sweep) and $R-R$ (downstream sweep) combinations in the ensemble averages lead to the overall positive character in cross-correlation curve 7. As seen, the curves 6 and 7 cross-correlation broad modes are more complex in overall shape than the already discussed cases; the variations may be more closely related to the $1-\mathrm{i}(\mathrm{u}) / 1-\mathrm{i}(\mathrm{d})$ or $1-\mathrm{ii}(\mathrm{u}) / 1-\mathrm{ii}(\mathrm{d})$ characteristic signatures correlating with the local maximum in $P_{i}$ near the fin root. The important point is that the overall maximum values in the cross-correlations agree with the qualitative models. The transition point in cross-correlation curve 3 has no broad mode since locally $\sigma_{\mathrm{Pw}}(x)$ is at a minimum so that the broad mode contribution to the overall $\sigma_{\mathrm{Pw}}(\mathrm{x})$ value is at a minimum (locally, the flattened portion of $P_{i}$ is translating over this region, resulting in the $\sigma_{\mathrm{Pw}}(\mathrm{x})$ minimum values); the transition point in cross-correlation curve 5 has a positive maximum and a negative maximum, suggesting that the station $-3.5 \xi$ data contains information from the stations just upstream and just downstream of it, similar to what was seen in the curve 5 ensemble averages.

In cross-correlation curves 1 through 4 (row (c)) the sharp mode always has a positive valued maximum. This can be attributed to the convection of a given signature shape through the interaction such that the same signal signature shape is detected at successive downstream stations (see curves 1-i(u) and 1-i(d) and the sharp signatures in the curves 2 and 3 ensemble averages resulting in a P-P or T-T behavior). It is not clear if this behavior persists beyond station $-4.5 \xi$ (this station might represent the downstream end of the primary horseshoe vortex so that the reversed flow may possibly not occur beyond this location). However, the RSED result suggests the possible presence of this effect. For the 
transition point of cross-correlation curve 3 only the sharp mode is present. Again, this is probably due to the fact that this station is at a local minimum in the $\sigma_{\mathrm{Pw}}(\mathrm{x})$ distribution indicating that the broad mode fluctuations are also at a local minimum so that only the sharp mode is evident.

\section{Analysis C Discussion}

For the cross-correlation results of analysis C (row (d) of Table 4) the discussion follows essentially the same lines as for analysis A, starting with the broad modes of the non-transition points. Cross-correlation curve 1 of analysis $C$ is the opposite of curve 2 of analysis $A$ since for the latter the undisturbed flow transducer was the reference and since for the former the downstream transducer was the reference. The same ensemble average relationship between the measurement stations $(R-F, F-R)$ of course holds for this analysis $C$ result as discussed for analysis A. For cross-correlation curves 2 and 6 (row (d)), the positive maxima occur since the ensemble averages are of a R-R or F-F nature. Further, the negative maxima of cross-correlation curves 4 and 7 can be related to the R-F/F-R behavior in the curves 2 and 4, and curves 2 and 7 ensemble averages. Curves 6 and 7 are also of a more complicated nature, as in analysis A.

The sharp mode behavior in analysis $\mathrm{C}$ arises in the same way as discussed above for analysis A. It is evident in curves 1 through 4 of row (d) (weakly in 2) but is not as well defined as before. This is probably due to the fact that for analysis $A$ the upstream transducer predominantly detects sharp mode fluctuations which, for case $\mathrm{C}$, are dominated in magnitude by the broad mode. The sharp mode existence in curves 5 through 7 is again unclear; the RSED calculations suggest their possible occurrence. 
The transition point indicated by cross-correlation curve 3 (row (d)) has characteristics similar to those of curves 2 and 4 . The flattened portion for time $\tau>0$ is the beginning of a rapid transition to the shape in curve 4 (see Brusniak (1993) for full details). Also, curve 5 is similar to curve 6 , suggesting the presence of the signal from the curve 6 region.

The trends in the cross-correlations of Dolling and Brusniak (1991) are the same as the cross-correlations presented here for analysis C. As such, the same conclusions will probably apply to the Dolling and Brusniak (1991) results. The strong cross-correlation and coherence results between the intermittent channel and the channel located at the second local maximum in the $\sigma_{\mathrm{Pw}}(\mathrm{x})$ distribution are seen to be due to large broad mode contributions from $P_{i}$ to the signals measured at the respective stations since $\sigma_{\mathrm{Pw}}(\mathrm{x})$ is maximum at the latter station (this also explains why the separated region channel had a low frequency contribution in the power spectrum in the same frequency range as the intermittent region spectra: both stations were located under portions of $P_{i}$ for which $\partial P_{i} / \partial \mathrm{x}$ was maximum). The cross-correlations were weak for the separated flow channel located further upstream since this is where $\sigma_{\mathrm{Pw}}(\mathrm{x})$ is smallest and where the local segment of $P_{i}$ tends to be flat.

\section{Analysis D Discussion}

For analysis D, row (e) of Table 4, all stations were at a $1 \xi$ separation distance so that the expectation is that all cross-correlations would be positive valued, since the signals are very similar for both stations (R-R, F-F, P-P, T-T behavior); the exception of course being when the two stations are in regions for which the two signals are dissimilar. From Table 4, 
row (e), it is seen that all of the cross-correlation curves except curve 7 are entirely positive valued. Curve 1 gives a standard cross-correlation for the undisturbed flow component and has only a sharp mode. In curve 2 the broad mode dominates such that the sharp mode can barely be detected. For curve 3, $\sigma_{\mathrm{PW}}(\mathrm{x})$ has reached a local minimum; because of this the broad mode width is also a minimum, as discussed in an earlier section, and the sharp mode stands out clearly. For curve $4, \sigma_{\mathrm{Pw}}(\mathrm{x})$ is at a local maximum so that the broad mode variations are now at a local maximum and the broad mode width is also a local maximum. The $\sigma_{\mathrm{Pw}}(\mathrm{x})$ distribution is again near a local minimum for the curve 5 result to the point that the broad mode is essentially absent (the end of the $P_{i}$ curve near station $-4.5 \xi$ is apparently flat so that broad mode variations will be small). A broad mode dominates again for curve 6 . A sharp mode apparently still exists in curve 6 . The positive/negative valued character of curve 7 is likely due to the R-F and F-R behavior in the ensemble averages as well as a R-R and F-F behavior still likely due to the close spacing between transducers.

\section{Additional Remarks}

By referring to Table 3 and Fig 8 it is now clear that the transition points from crosscorrelation analyses B and C correspond to the stations centered near $-14.0 \xi,-4.0 \xi$ and $-1.0 \xi$, for which the cross-correlation coefficient changes sign and also for which the ensemble averages record the passage of a local maximum or minimum in $P_{i}$. The analysis D crosscorrelation transition points are also related to the passage of the local maxima and minima in $P_{i}$, where the station $-9.0 \xi$ transition point was seen to be due to the low frequency fluctuations contributing to the local maximum in $\sigma_{\mathrm{Pw}}(\mathrm{x})$. The transition points from analysis $\mathrm{A}$ are the same as all the other cases, whereas the source of the correlations is not due exclusively to the presence of $P_{i}$. 


\section{Validation of Flowfield Model Through Prediction of Shock Foot Motion}

From the preceding section it was seen that the ensemble averages had essentially both a broad and a sharp signature; similarly, the cross-correlations had typically a broad mode and a sharp mode. The sharp signature of the ensemble average and sharp mode of the cross-correlation were attributed to the convection of the initially undisturbed incoming turbulent boundary layer through the interaction. From the ensemble average wall pressure distributions at $\tau=0$ the hypothesis that a "self-similar" pressure distribution, $P_{i}$, was responsible for the measured flowfield properties downstream of UI was proposed and investigated. From the model the $\sigma_{\mathrm{Pw}}(\mathrm{x})$ shape was predicted accurately, the ensemble averages were related logically to local segments of $P_{i}$, and the cross-correlations were explained as being ultimately due to $P_{i}$ in terms of qualitative cross-correlation models. The $P_{i}$ model agreed well with the broad signatures of the ensemble averages and broad modes of the cross-correlations.

The ensemble averages used have so far not distinguished between different types of shock sweep motions which include short sweeps, which occur over only 1 transducer before the shock turns around, and long sweeps, which can occur successively over several transducers. All of these cases are included in the ensemble average results, so that no distinction exists between low frequency oscillations and high frequency "jitter" motion of the shock foot. The question then is whether the $P_{i}$ model is accurate only in a timeaveraged sense or if it is representative at each instant in time as well. One way to approach this question is to ask the following: if the wall pressure at a given point under the separated 
region is due to an instantaneous, "self-similar" $P_{i}$ distribution, can the separated region pressure values at each instant in time be used to predict the instantaneous location of the separation shock foot? This would check whether the separated region pressure levels correlate with shock foot location in an instantaneous as well as time-averaged sense. The approach is as follows: first, obtain the shock foot time history, $X_{s}(t)$, directly using the intermittent wall pressure data; second, use the separated region pressure signals to obtain the predicted shock foot time history, $x_{s}(t)$; then, use basic statistical analysis and spectral analyses to compare the two results.

The procedure used to obtain $x_{s}(t)$ is as follows (the procedure for obtaining $X_{s}(t)$ was described in the 'Experimental Program and Analysis Techniques' section). For this case, four transducers were located in the intermittent region from $-16.5 \xi$ to $-19.5 \xi$, spanning an intermittency range of 0.9 to 0.25 , respectively. Although this corresponds to only about $50 \%$ of the total range of the shock foot motion in physical space, it encompasses $65 \%$ of the intermittent region (i.e., a majority of the shock foot motion could be monitored). The second set of four transducers was located in the separated region from $-8.5 \xi$ to $-11.5 \xi$, all well downstream of 'S'. First, ensemble averages for both upstream and downstream sweeps were obtained, from which the separated region ensemble average pressures at time $\tau=0$ versus shock foot location were obtained (Table 5). Next, for each separated region station a least squares curve fit between shock foot location and ensemble averaged pressure was obtained, producing the calibration curves in Table 5; by doing this separately for each channel, effects of transducer zero drift could be removed. Finally, $x_{s}(t)$ at each instant in time was obtained by calculating four $x_{s}(t)$ values from each of the separated region pressure-time values using the curve fits and then averaging the four $x_{S}$ values to produce a single weighted estimate for $x_{s}(t)$ at the given time instant. Although the calibration was 
done only for the shock foot range of $-16.5 \xi$ to $-19.5 \xi$, the extrapolated values of $x_{s}(t)$ were retained in the prediction.

Fig 18 shows simultaneous samples of both $X_{s}(t)$ and the $x_{s}(t)$ shock foot history obtained using the above approach. The $X_{s}(t)$ signal displays a low frequency trace; $x_{s}(t)$ exhibits both low and high frequency variations. From a statistical analysis of $x_{s}(t)$ the mean location was calculated to be at stations $-18.24 \xi$ and $-18.77 \xi$ for the upstream sweep and downstream sweep calibration curves, respectively. This is in close agreement with the station at which the measured value of $\gamma$ is equal to 0.5 , station $-18.5 \xi$. From the previous wall pressure measurements the intermittent region spans the region $-15.0 \xi$ to about $-23.5 \xi$. The $x_{s}(t)$ result, based on the extrapolated data, predicts an intermittent region spanning about $-12.1 \xi$ to $-24.4 \xi$ and about $-12.0 \xi$ to $-25.5 \xi$ for the upstream and downstream sweep cases, respectively, based on a $\bar{x}_{s}(t) \pm 3 \sigma_{x_{s}(t)}$ variation. By initially lowpass digitally filtering the data prior to calculating $x_{s}(t)$, the intermittent region upstream end was calculated to be about $1 \xi$ further downstream and the downstream end was calculated to be at about $-13.0 \xi$ for both cases. An improved result would probably be obtained by having the intermittent region pressure transducers span the entire intermittent region for a more accurate calibration so that extrapolation does not occur (however, this can be difficult for large interactions).

Power spectra of $X_{S}(t)$ and the unfiltered $x_{s}(t)$ result are shown in Fig 19 (upstream sweep case; the downstream sweep case is essentially the same). As seen, the $X_{s}(t)$ spectrum is low frequency dominated, whereas the $x_{s}(t)$ spectrum exists up to the $50 \mathrm{kHz}$ analog filter limit. The most striking feature is the similarity of the two results for frequencies below about $2 \mathrm{kHz}$. In fact, the well defined peak at about $400 \mathrm{~Hz}$ from $\mathrm{X}_{\mathrm{s}}(\mathrm{t})$ is also detected in 
$x_{s}(t)$; the additional $X_{s}(t)$ peaks at about $750 \mathrm{~Hz}$ and $1 \mathrm{kHz}$ are detected as well. Beyond the $2 \mathrm{kHz}$ range the $x_{s}(t)$ result exhibits a significant amount of energy in the 10 to $30 \mathrm{kHz}$ range. The striking similarities at low frequency between the $X_{s}(t)$ and $x_{s}(t)$ spectra confirms that the $x_{s}(t)$ prediction procedure has succeeded in reproducing the low frequency component of the separation shock foot motion and that the $P_{i}$ distribution is a physically meaningful instantaneous model. This can even be seen in Fig 18, where the low frequency component of $x_{s}(t)$ does essentially match $x_{s}(t)$. The $x_{s}(t)$ prediction of a high frequency band of energy is somewhat of an enigma. Presently no direct measurements of the high frequency motion of the shock foot are available for comparison with the $x_{s}(t)$ result. As a consequence, it is not known if the energy band is a true representation of shock foot motion or stems from some other source such as incoming undisturbed flow fluctuations being detected by the separated region transducers as the separated shear layer passes above and is manifested in $x_{s}(t)$ as a high frequency shock foot motion.

The cross-correlation of $\mathrm{X}_{s}(t)$ with $\mathrm{x}_{s}(t)$ is shown in Fig 20 (upstream sweep case; the downstream sweep case is essentially the same). It is characterized by a positive valued peak occurring at $-30 \mu \mathrm{s}$. The strong correlation maximum (larger than 0.65 ) again demonstrates the ability of the technique to predict the separation shock foot motion. In obtaining $x_{s}(t)$ the separated region pressure levels at each instant in time were used to predict an instantaneous shock foot location. The fact that the cross-correlation peak occurs at negative time delay suggests that the separated region pressure fluctuations precede separation shock foot motion (i.e., that the separated region causes the shock motion). For the lowpass digital filtering case the cross-correlation is essentially the same, except that the maximum value is now larger than 0.75 . 
Finally, the coherence between $X_{s}(t)$ and $x_{s}(t)$ (upstream sweep case) is shown in Fig 21. The strong coherence for frequencies less than about $1 \mathrm{kHz}$ demonstrates that the $X_{s}(t)$ and $x_{s}(t)$ time histories are related in a highly linear manner for those frequencies.

\section{Brief Comments on Practical Implications of Results}

\section{Computational Fluid Dynamics}

Fig 22 shows the mean wall pressure distribution (compared with the experiments of Dolling, et al. (1979) and Dolling and Bogdonoff (1982)) and particle paths in the plane of symmetry from the Mach 3 blunt fin computation by Hung and Buning (1985). The mean wall pressure distribution, for $\delta / \mathrm{D}=1.0$, agrees well with the experimental data (for $\delta / \mathrm{D}=$ 0.26 the agreement is not as good, although reasonable overall). The inset shows the peak near the root for $\delta / D=0.26$, which agrees with the results presented in this paper for which $\delta / D$ is close to 1 . In particular, this root peak value was seen in the ensemble average result of curves 6, Fig 10 (see also the $P_{i}$ sketch at the top of Fig 16$)$. The particle paths $(\delta / \mathrm{D}=$ 1.0) show a primary horseshoe vortex about $1.5 \mathrm{D}$ in length with its core about $0.2 \mathrm{D}$ above the surface, as well as a small secondary vortex near the fin root. The pressure minimum for $\mathrm{x}>-1.5 \mathrm{D}$ is associated with a reversed high speed flow zone attributed to an "image or socalled ground effect of the vortex." In terms of the experimental and computational results the effect of flow unsteadiness on the horseshoe vortex can now be inferred. In particular, the shape of the $P_{i}$ distribution (the minimum) is inferred to be due to the vortex ground effect, as caused by the instantaneous vortex shape. Thus, as the separation shock foot moves upstream and downstream, the vortex expands and contracts and consequently weakens and strengthens, so that the centerline pressure variations are due to a time-varying 
$P_{i}$ distribution which undergoes aperiodic motion.

It appears, then, that the role of the model geometry is as follows: first, the model geometry establishes the "steady" flowfield character (horseshoe vortex) which gives rise to a given $P_{i}$ distribution; then, the addition of turbulence is manifested as an unsteady flowfield in which the vortex oscillates aperiodically. A valid question is whether the vortex responds to the turbulence and consequently drives the flow, or whether the separation shock foot is driven by the turbulence and the vortex responds to the shock foot motion. The crosscorrelations between the intermittent region and separated flow region, as well as between $X_{s}(t)$ and $x_{s}(t)$, support the former, as does the recent work of Erengil and Dolling (1993).

As seen, the computation predicted a single result, although it is now clear that local measurements are actually due to a $P_{i}$ distribution, the front end of which translates aperiodically between UI and ' $S$ ' and undergoes distortion. The question, then, is, does the computational solution $\left(\overline{\mathrm{P}}_{\mathrm{w}}(\mathrm{x})\right.$, Fig 22a) correspond to a particular $P_{i}$ distribution? If true, then could a series of $P_{i}$ distributions be calculated by solving for the flowfield with different incoming boundary conditions such that the separation shock foot was fixed at several stations spanning the intermittent region? From these, it would be theoretically possible to estimate loading levels (or at least determine where the maximum values would occur) as was done in obtaining the $|\Delta \mathrm{P}|$ distribution of Fig 14. All that would be needed for such calculations would be correlations of UI and ' $S$ ' locations. If the method is accurate, then dynamic solutions for other model geometries could be obtained from UI/'S' correlations entirely. This method would represent a first order inclusion of the flowfield unsteadiness into computations. 
To illustrate this idea the computation shown in Fig $22 \mathrm{a}$ for $\delta / \mathrm{D}=1$ (which was taken directly from Hung and Buning (1985)) has been digitized and is shown replotted in Fig 23a. In both the computation and the experiments additional tests were made with the same incoming flow conditions, but with a thinner boundary layer. The measured and computed wall pressures are shown in Fig 23b. There is again good agreement between computation and experiment. As seen, by changing the incoming boundary conditions (i.e., by decreasing the boundary layer thickness), the upstream influence location has been shifted downstream by about $0.3 \mathrm{D}$ (see Figs $23 \mathrm{a}$ and $23 \mathrm{~b}$ ). Since this length scale change is typical of the shock foot displacement in a given flow, these two data sets offer an opportunity to make a qualitative assessment of the ideas outlined above. The basic idea is that the pressure distribution of Fig $23 \mathrm{~b}$ is assumed to be the ensemble averaged $P_{i}$ distribution which would occur for the conditions of Fig 23a with the shock foot at the downstream end of the intermittent region. The pressure distribution in Fig $23 \mathrm{a}$ is assumed to be the $P_{i}$ distribution which occurs with the shock foot at the upstream end of the intermittent region. Fig $23 \mathrm{c}$ shows the $\mid \triangle \mathrm{Pl}$ distribution obtained from the two digitized computational results and Fig $23 \mathrm{~d}$ shows the measured standard deviation distribution. The computational result (Fig 23c) predicts three maximum values, near $-2.5 \mathrm{D},-1.5 \mathrm{D}$ and $-0.25 \mathrm{D}$ and minimum values near $-2 \mathrm{D}$ and at about $-0.5 \mathrm{D}$ to $-1 \mathrm{D}$. (Note. The distribution does not extend beyond $-2.5 \mathrm{D}$ since the experimental data shown in Hung and Buning (1985) cover the data from the computation). These agree qualitatively with the three maximum values in Fig $23 \mathrm{~d}$ near $-2.5 \mathrm{D},-1.25 \mathrm{D}$ and $-0.25 \mathrm{D}$, as well as the minimum values near $-2 \mathrm{D}$ and $-0.5 \mathrm{D}$. This example once more brings out the fact that the shape of the standard deviation distribution is due to displacement of a $P_{i}$ distribution. In addition to the computational result, Fig $23 \mathrm{c}$ also shows the $|\Delta \mathrm{P}|$ distribution obtained from the experimental data of Figs $23 \mathrm{a}$ and $23 \mathrm{~b}$. This $\mid \Delta \mathrm{PI}$ distribution also has three maximum values which occur at about $-2.25 \mathrm{D},-1.25 \mathrm{D}$ and $-0.25 \mathrm{D}$ as well as minimum 
values at about $-2 \mathrm{D}$ and $-0.5 \mathrm{D}$. The distribution does quite well in predicting the fluctuating pressure load distribution in terms of shape as well as magnitude, even though it is based on mean measurements alone.

The $I \Delta \mathrm{PI}$ distributions of Fig $23 \mathrm{c}$ (which were obtained from the computations and experiments), illustrate the concept and re-emphasize the possibility of using either computations or simple mean wall pressure measurements alone in order to obtain qualitative estimates of fluctuating pressure load distributions. In an experimental program for which a large variety of model geometries are available for fluctuating pressure measurements, the utility of the simplified approach becomes clear. In particular, the mean pressure distributions for all model geometries (for different boundary layer thicknesses) could be obtained, providing standard deviation distribution estimates for all cases. From these estimates the best candidates for intensive instantaneous measurements could be selected, thereby reducing the cost of the program.

A final point about Fig 23 is that changes in boundary layer thickness were used to generate the different mean wall pressure distributions, which in turn were used to estimate the wall pressure standard deviation distribution. Since the estimates provided reasonable results, this leads to the question of whether the unsteadiness of the flowfield is ultimately due to variations of the "short-term time-averaged" boundary layer height, $\delta_{i}$, with time. If true, then the instantaneous pressure distribution, $P_{i}$, would depend on the character of $\delta_{i}$, such that when $\delta_{i}$ is small, the upstream extent of $P_{i}$ is small, and when $\delta_{i}$ is large, the upstream extent of $P_{i}$ is large. This makes physical sense since when $\delta_{i}$ is small the boundary layer mass entering the horseshoe vortex will be small and the vortex itself will be small and, when $\delta_{i}$ is large the entering boundary layer mass will be large and the horseshoe 
vortex will be large.

\section{Flow Control}

It was seen earlier that the large amplitude low frequency fluctuations which dominate the standard deviation distribution result from $P_{i}$ and are related to the pressure difference $|\Delta \mathrm{P}|$ due to a translating $P_{i}$ distribution. Therefore, in order to reduce the amplitude of local fluctuations the local $|\Delta \mathrm{P}|$ variation, which occurs at low frequency, needs to be minimized. At this stage it does not appear that altering the high frequency content would be productive. Methods need to be found which reduce the magnitude of the $P_{i}$ gradient $\left(\partial P_{i} / \partial \mathrm{x}\right)$ so that $|\triangle \mathrm{P}|$ is minimized. Since $P_{i}$ is due to the horseshoe vortex it may be possible to alter the large scale low frequency fluctuations by manipulating this vortex through model geometry changes. Since $P_{i}$ and the mean pressure distribution have similar shapes, measurements of $\bar{P}_{w}(x)$ alone may be sufficient to judge the effectiveness of a given method, as discussed above. 


\section{Summary and Conclusions}

Fluctuating wall pressure measurements have been made on centerline upstream of a blunt fin in a Mach 5 turbulent boundary layer. Experiments were made in which a reference transducer was located under the undisturbed incoming turbulent boundary layer and simultaneous measurements were made at successive locations downstream of this station (analysis A). Analyses B and C were essentially the same as analysis A, but the reference transducer was located at different stations in the intermittent region. Pairs of transducers at minimum spacing $(0.115 \mathrm{in}$.) were also positioned throughout the centerline flowfield in order to study convective phenomena. Cross-correlation results from these analyses demonstrated that a correlation does exist between each of the three flowfield regions. The cross-correlation results showed the general existence of two modes, a broad mode associated with the low frequency content of the signals, and a sharp mode associated with high frequencies. "Transition" points in the flowfield were identified at which the crosscorrelations changed character. Ensemble averages of the $\mathrm{B}$ and $\mathrm{C}$ test series showed the existence of two characteristic signatures, a broad (low frequency) signature and a sharp (high frequency) signature. Digital filtering demonstrated that frequencies below $4 \mathrm{kHz}$ contributed to the low frequency (broad mode) cross-correlation, and also that the ensemble averages contained low frequency contributions which were not obvious from the unfiltered result.

By examining the ensemble average wall pressure distributions for different shock foot fixed positions, it has been shown that local fluctuating wall pressure measurements are due to a distinct pressure distribution, $P_{i}$, which undergoes a stretching and flattening effect 
as its upstream boundary translates aperiodically between the upstream influence and separation lines. The locations of the maxima and minima in the centerline wall standard deviation distribution were accurately predicted using this distribution, providing quantitative confirmation of the model. A qualitative discussion of the local wall pressure measurements which would be obtained if $P_{i}$ was shifted upstream or downstream, analogous to upstream and downstream sweeps of the shock foot, agreed well with the observed character of the ensemble averages. Further, by a qualitative examination of the type of cross-correlations which would be obtained from certain model signals, and by then comparing the ensemble average results to the model signals, the source of the broad mode in the cross-correlations was qualitatively verified. It was seen that the low frequency content of the pressure signals from downstream of the shock foot as well as the corresponding broad mode and broad signature of the cross-correlations and ensemble averages are due to the unsteady $P_{i}$ distribution. In addition, the high frequency content of the signals, as well as the corresponding sharp mode of the cross-correlations and sharp signature in the ensemble averages are due to convection of the incoming undisturbed boundary layer flow into the interaction.

Additional quantitative support for the $P_{i}$ model was provided when wall pressure signals from under the separated flow region were used to predict the position-time history of the separation shock foot. The low frequency content of the predicted shock foot positiontime history, $x_{s}(t)$, matched extremely well with the measured shock foot position-time history, $X_{s}(t)$ (for frequencies less than about $2 \mathrm{kHz}$ ). The method also predicted a shock motion in the 10 to $30 \mathrm{kHz}$ range which could not be confirmed, since no measurements of separation shock foot motion for frequencies greater than about $2 \mathrm{kHz}$ are available for comparison with $x_{s}(t)$. The negative time delay peak in the cross-correlation between $x_{s}(t)$ 
and $X_{s}(t)$ suggests that the separated region fluctuations precede shock foot motion, as does Erengil and Dolling (1993).

Finally, the unsteady flowfield can be described as follows: The model geometry establishes the "steady" flowfield character, namely the horseshoe vortex (and secondary root vortex) which gives rise to the "steady" $P_{i}$ distribution. With turbulence the flowfield becomes unsteady. In particular, as the foot of the separation shock moves upstream and downstream the leading edge of the vortex also moves upstream and downstream, and the vortex expands and contracts (and consequently weakens and strengthens), so that the centerline pressure variations are due to a time-varying $P_{i}$ distribution which undergoes distortion and aperiodic motion. The results lead to the following implications. First, in order to minimize the fluctuating loads caused by the flow unsteadiness, methods should focus on reducing the magnitude of the $P_{i}$ gradient $\left(\partial P_{i} / \partial \mathrm{x}\right)$. Second, by calculating pressure distributions for several shock foot fixed cases, in conjunction with shock foot length scale correlations and intermittency distributions, it may be possible to predict some of the unsteady aspects of shock wave/turbulent boundary layer interaction separated flowfields.

\section{Acknowledgements}

This work was supported by NASA Lewis under Grant NAG3-1023 monitored by Warren Hingst. This source of support is gratefully acknowledged. 


\section{References}

Barnhart, P. J. (1993), Private Communication.

Bendat, J. S. and Piersol, A. G. (1986), Random Data, Analysis and Measurement Procedures, 2nd Ed., John Wiley \& Sons, Inc., New York, 1986

Brusniak, L. (1993) "Flowfield Dynamics in Blunt Fin-Induced Shock Wave/Turbulent Boundary Layer Interactions," PhD Dissertation, Dept. of Aerospace Engineering and Engineering Mechanics, University of Texas at Austin, Fall 1993.

Dolling, D. S., Cosad, C. D., and Bogdonoff, S. M. (1979), "An Examination of Blunt FinInduced Shock Wave Turbulent Boundary Layer Interaction," AIAA Paper 79-0068, 17th Aerospace Sciences Meeting, New Orleans, LA, Jan. 15-17.

Dolling, D. S., and Bogdonoff, S. M. (1981,1), "Scaling of Interactions of Cylinders with Supersonic Turbulent Boundary Layers," AIAA Journal, Vol. 19, No. 5, May 1981, pp. 655-657.

Dolling, D. S. and Bogdonoff, S. M. (1981,2), "An Experimental Investigation of the Unsteady Behavior of Blunt Fin-Induced Shock Wave Turbulent Boundary Layer Interactions," AIAA Paper 81-1287, AIAA 14th Fluid \& Plasma Dynamics Conference, Palo Alto, CA, June 1981.

Dolling, D. S. and Bogdonoff, S. M. (1982), "Blunt Fin-Induced Shock Wave/Turbulent Boundary Layer Interaction," AIAA Journal, Vol. 20, No. 12, Dec. 1982, pp. 1674-1680.

Dolling, D. S. and Smith, D. R. (1989), "Separation Shock Dynamics in Mach 5 Turbulent Interactions Induced by Cylinders," AIAA Journal, Vol. 27, No. 12, Dec. 1989, pp. 16981706.

Dolling, D. S. and Brusniak, L. (1989), "Separation Shock Motion in Fin, Cylinder, and Compression Ramp-Induced Turbulent Interactions," AIAA Journal, Vol. 27, No. 6, June 1989, pp. 734-742.

Dolling, D.S. and Brusniak, L. (1991), "Correlation of Separation Shock Motion in a Cylinder-Induced Interaction with Pressure Fluctuations Under the Separated Region," 
Kleifges, K. and Dolling, D. S. (1993), "Control of Unsteady Shock-Induced Turbulent Boundary Layer Separation Upstream of Blunt Fins," AIAA Paper 93-3281, AIAA Shear Flow Conference, Orlando, FL, July 6-9, 1993.

Kussoy, M.L., Brown, J. D., Brown, J. L., Lockman, W. K., and Horstman, C. C., (1987) "Fluctuations and Massive Separation in Three-Dimensional Shock-Wave/BoundaryLayer Interactions," 2nd International Symposium of Transport Phenomena in Turbulent Flows, Tokyo, Japan, Oct. 25-29, 1987.

Lakshmanan, B. and Tiwari, S. N. (1993), "Study of Supersonic Intersection Flowfield at Modified Wing-Body Junctions," AIAA Journal, Vol. 31, No. 5, May 1993, pp. 877-883.

McClure, W.B. (1992), "An Experimental Study of the Driving Mechanism and Control of the Unsteady Shock Induced Turbulent Separation in a Mach 5 Compression Corner Flow," PhD Dissertation, Dept. of Aerospace Engineering and Engineering Mechanics, University of Texas at Austin, August 1992.

Narlo, J. C. (1986), "Experimental Investigation of the Driving Mechanisms of Separation Shock Wave Motion in Interactive Flows," M. S. Thesis, Dept. of Aerospace Engineering and Engineering Mechanics, University of Texas at Austin, Dec. 1986.

Pozefsky, P., Blevins, R. D., and Laganelli, A. L. (1989), "Thermo- Vibro-Acoustic Loads and Fatigue of Hypersonic Flight Vehicle Structures," AFWAL TR-89-3014, Feb. 1989.

Price, A. E. and Stallings, R. L. (1967), "Investigation of Turbulent Separated Flows in the Vicinity of Fin Type Protuberances at Supersonic Mach Numbers," NASA TN D-3804, Feb. 1967.

Sun, C.-C. and Childs, M. E. (1973), "A Modified Wall-Wake Velocity Profile for Turbulent Compressible Boundary Layers," Journal of Aircraft, Vol. 10, No. 6, pp. 381-383. 

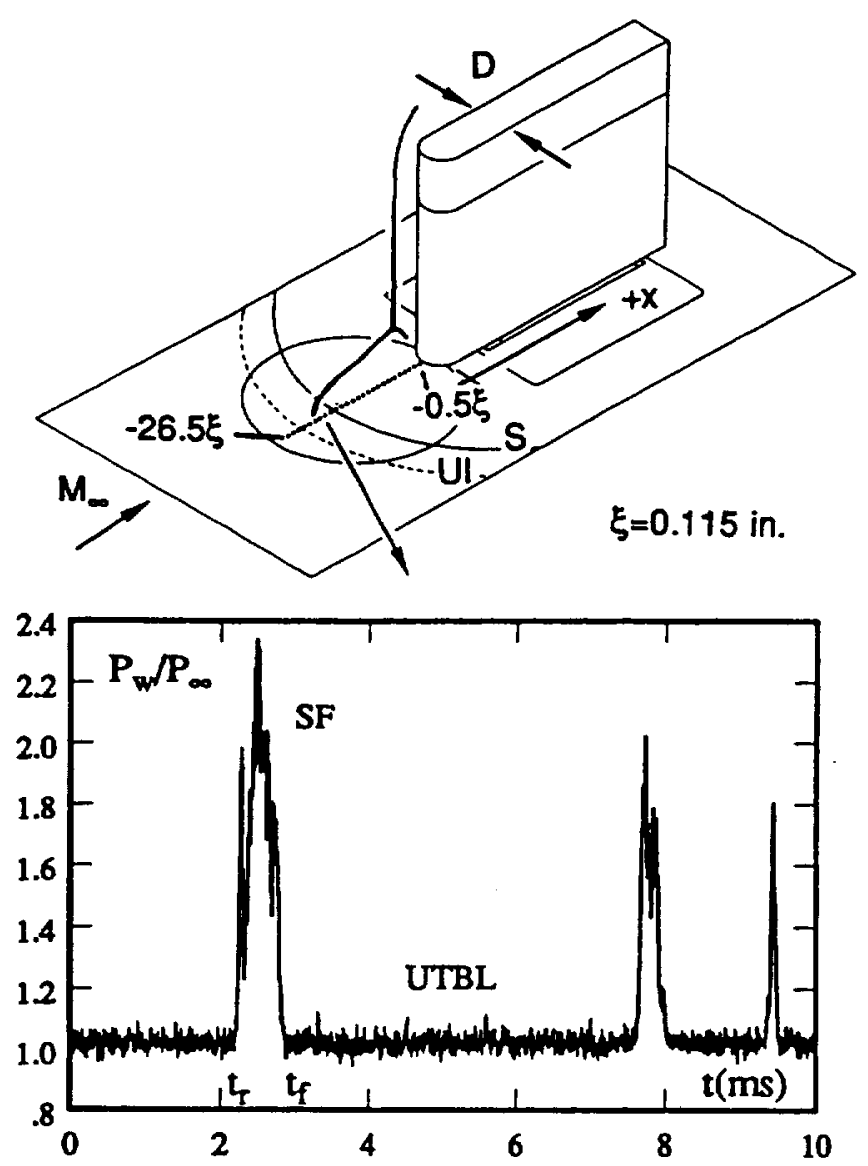

Fig 1 Blunt fin flowfield. 

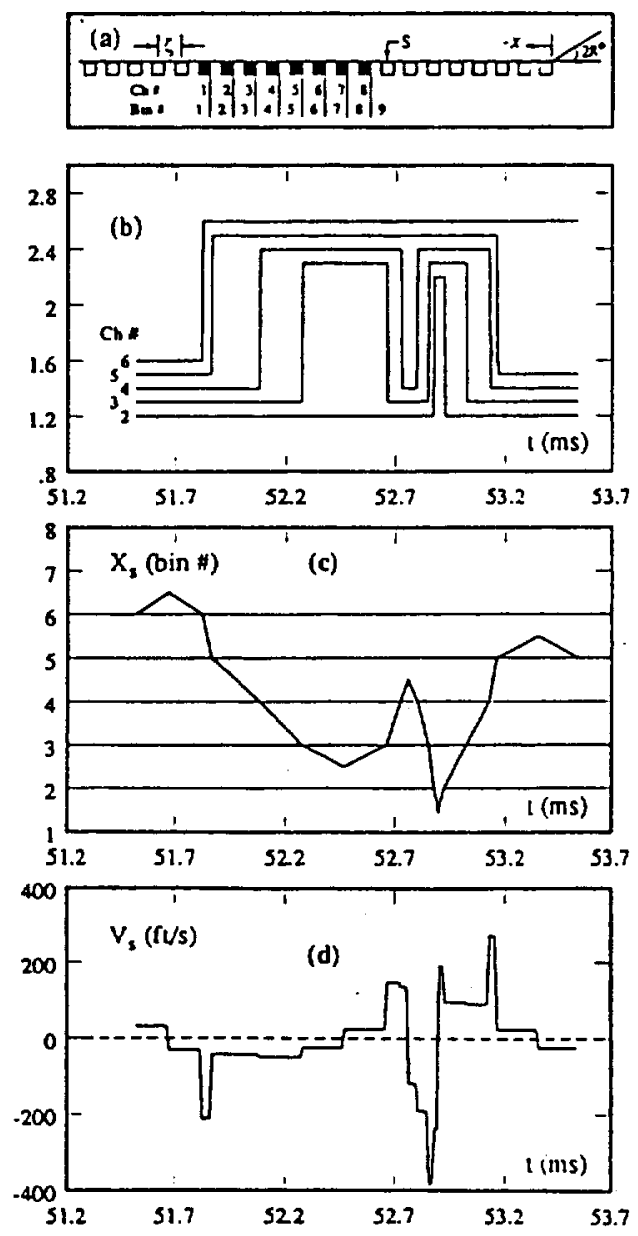

Fig 2 Conversion technique for separation shock foot position-time history (from Erengil and Dolling (1992)). 


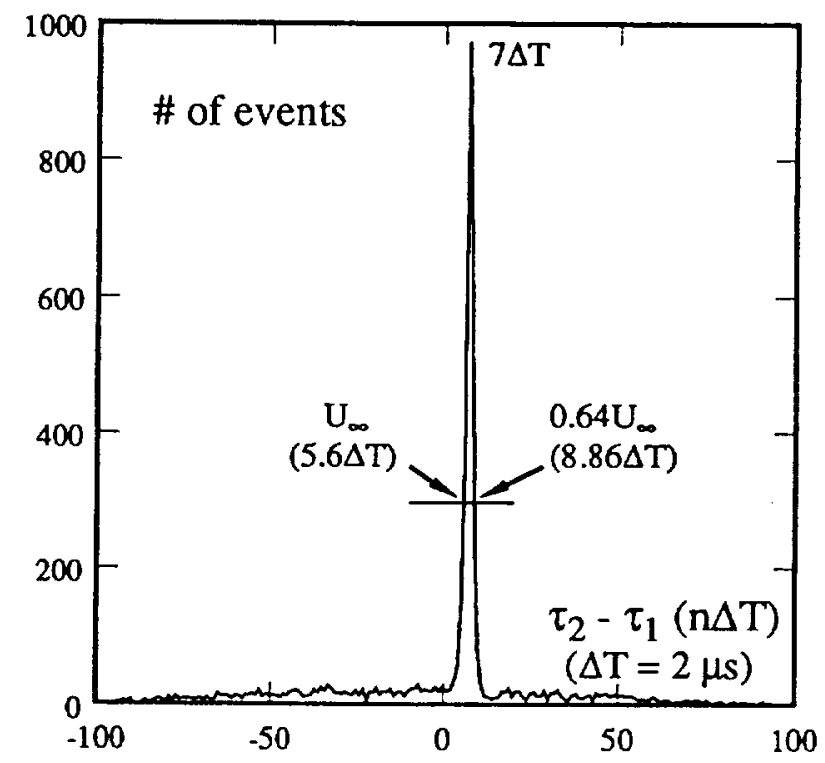

Fig 3 Example RSED histogram. 

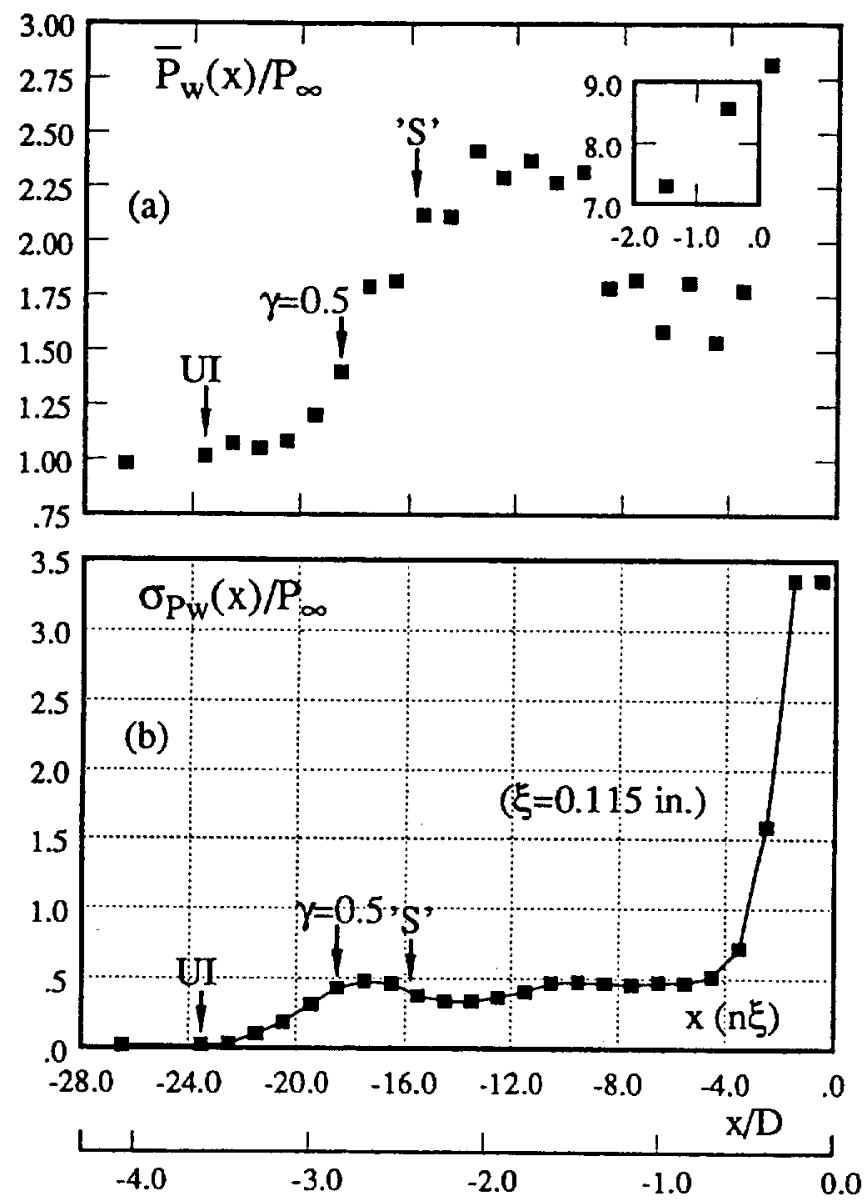

Fig 4 (a) Mean pressure distribution; (b) Standard deviation distribution. 

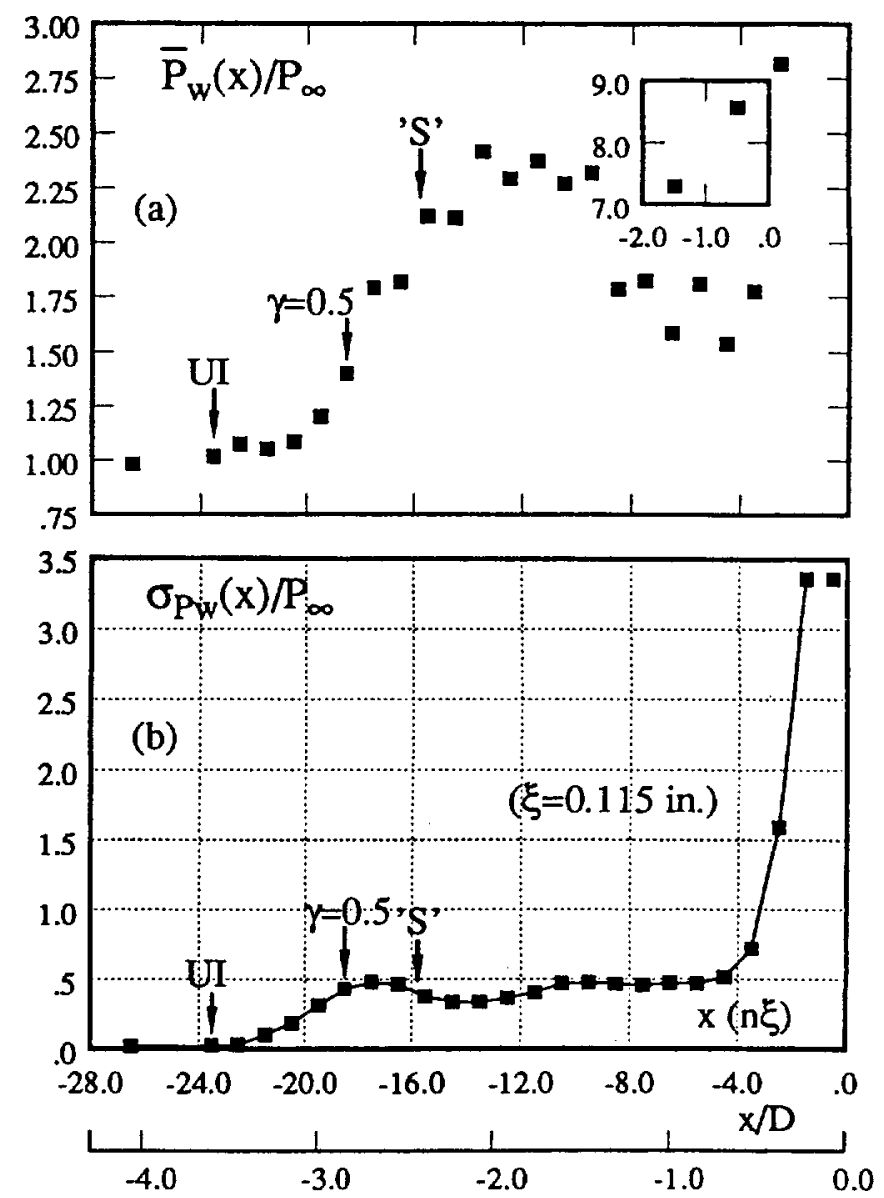

Fig 4 (a) Mean pressure distribution; (b) Standard deviation distribution. 


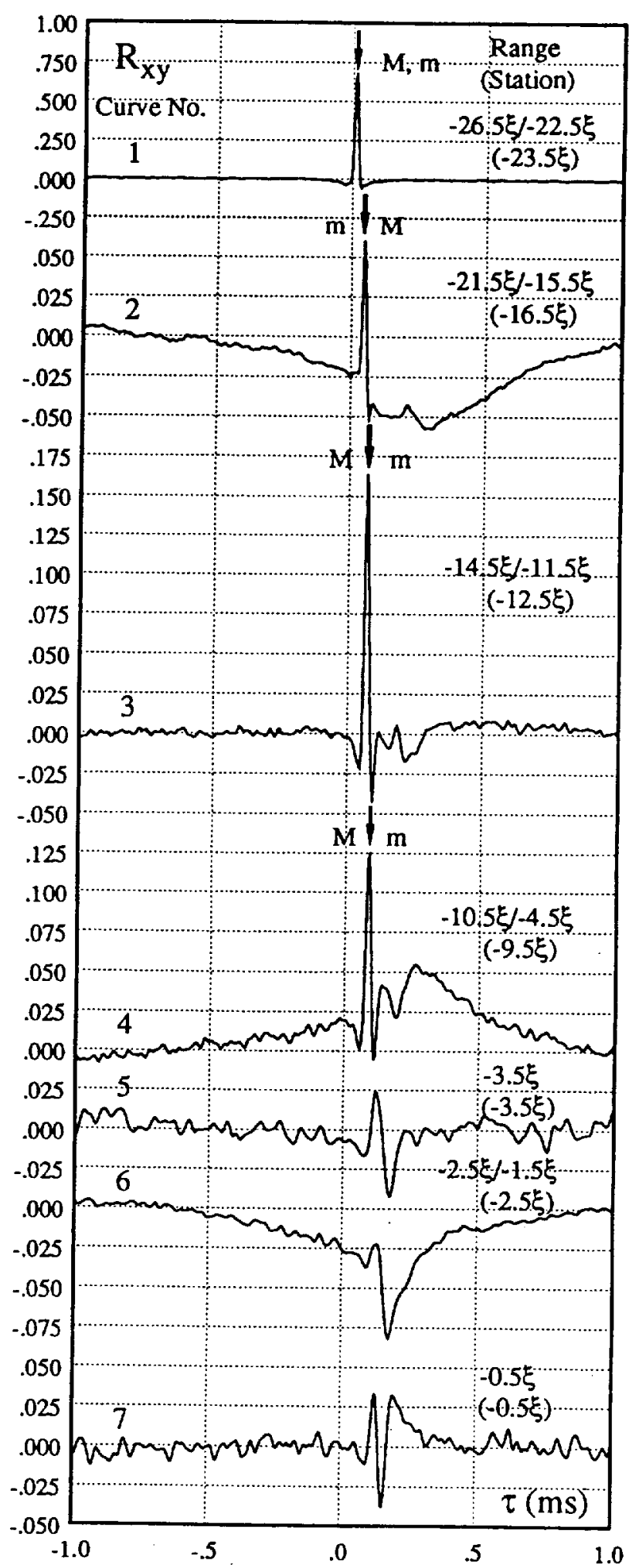

Fig 5 Analysis A cross-correlations. 


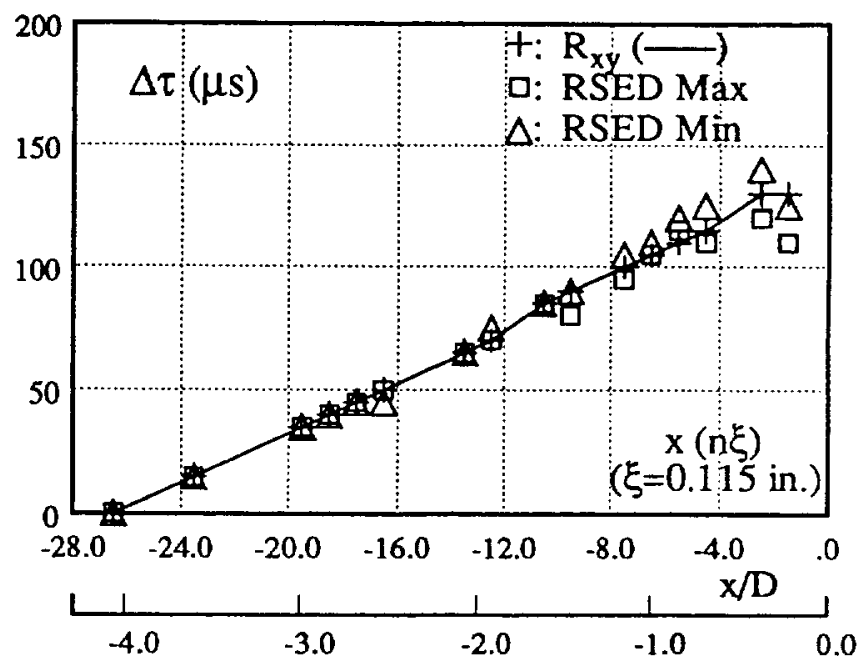

Fig 6 Time delay, analysis A. 


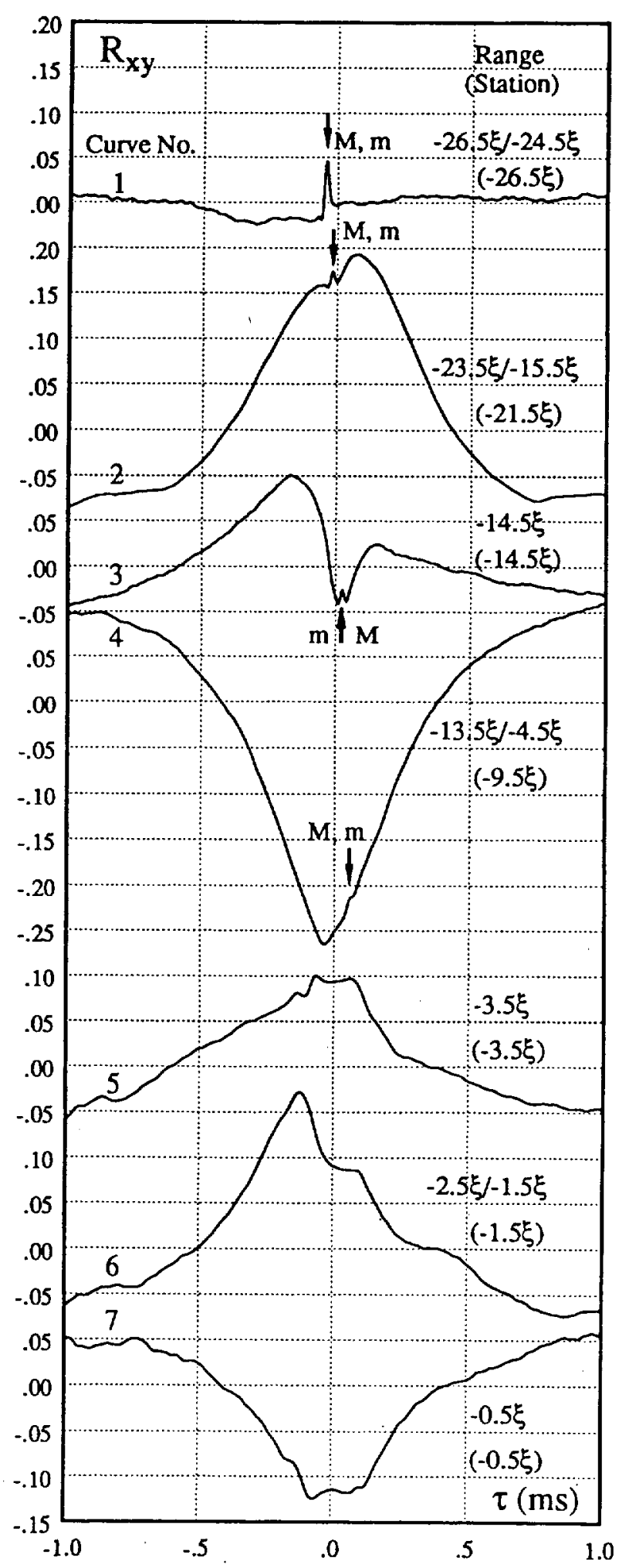

Fig 7 Analysis C cross-correlations. 


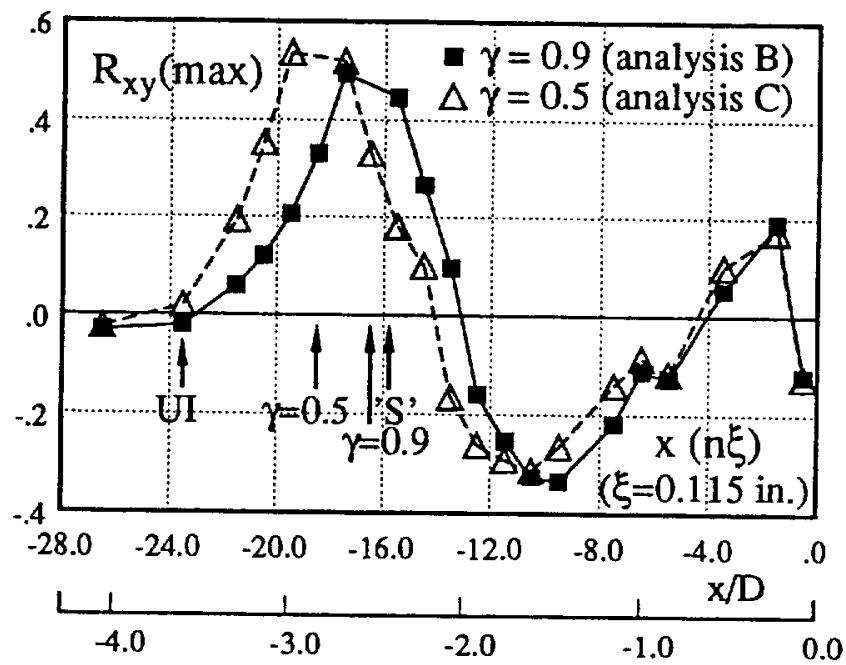

Fig 8 Analysis $\mathrm{C}$ cross-correlation broad mode (maximum) distribution. 


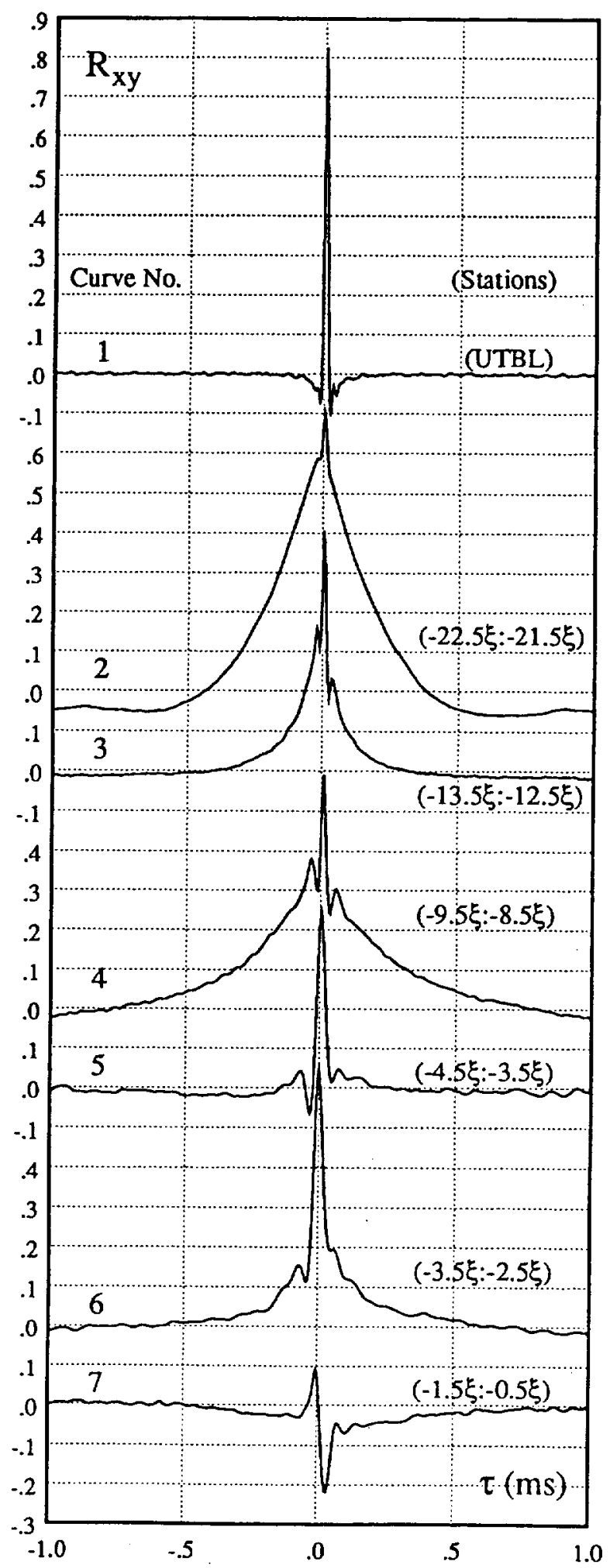

Fig 9 Analysis D cross-correlations. 


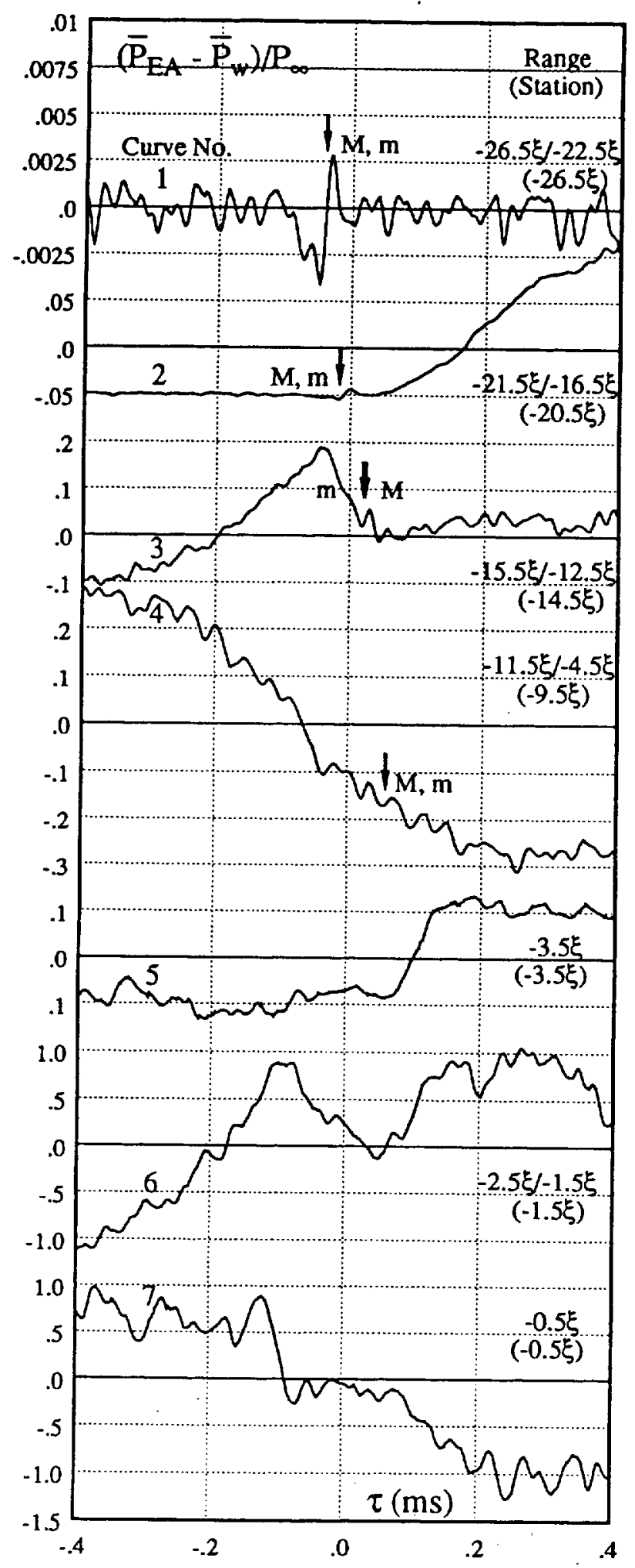

Fig 10(u) Analysis C upstream sweep ensemble averages.

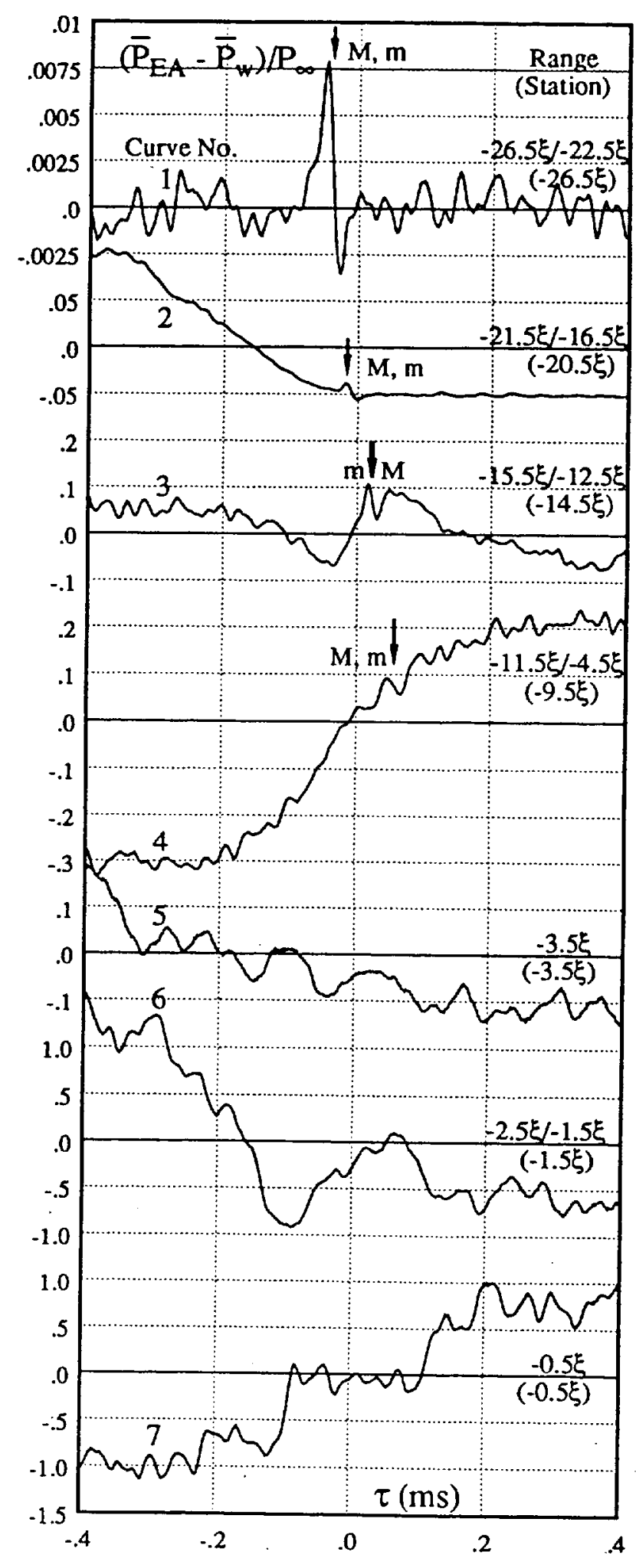

Fig 10(d) Analysis C downstream sweep ensemble averages. 


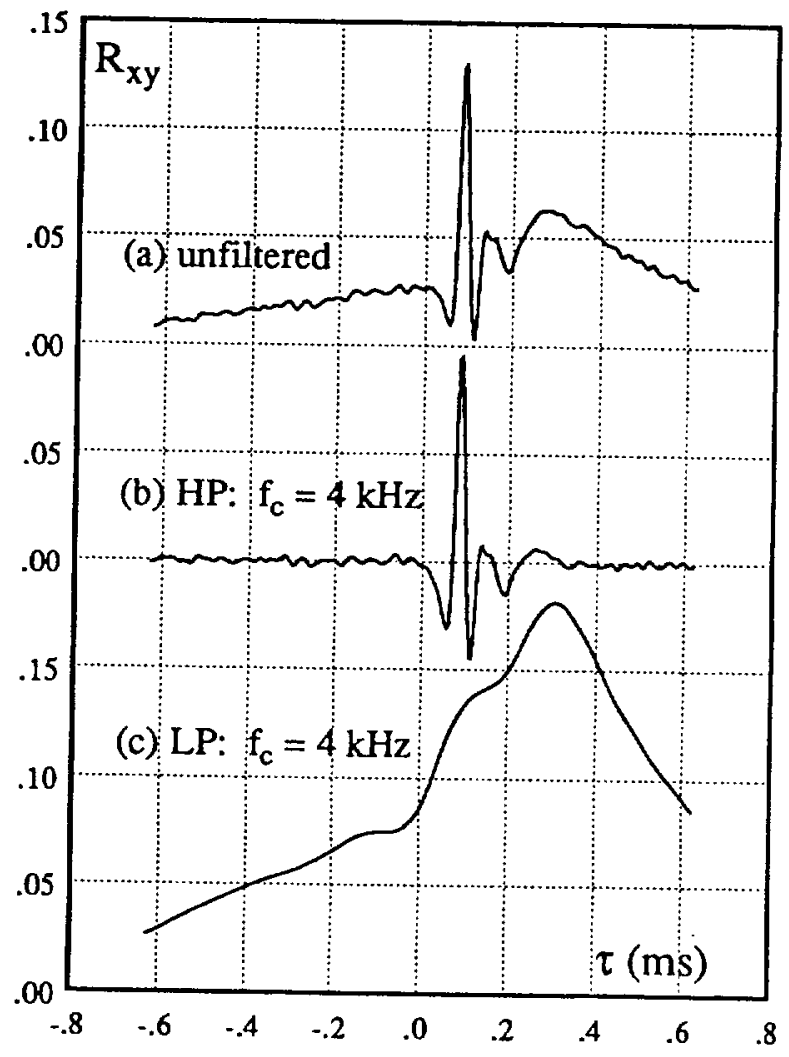

Fig 11 Digital filtering of incoming flow/separated region cross-correlation. 


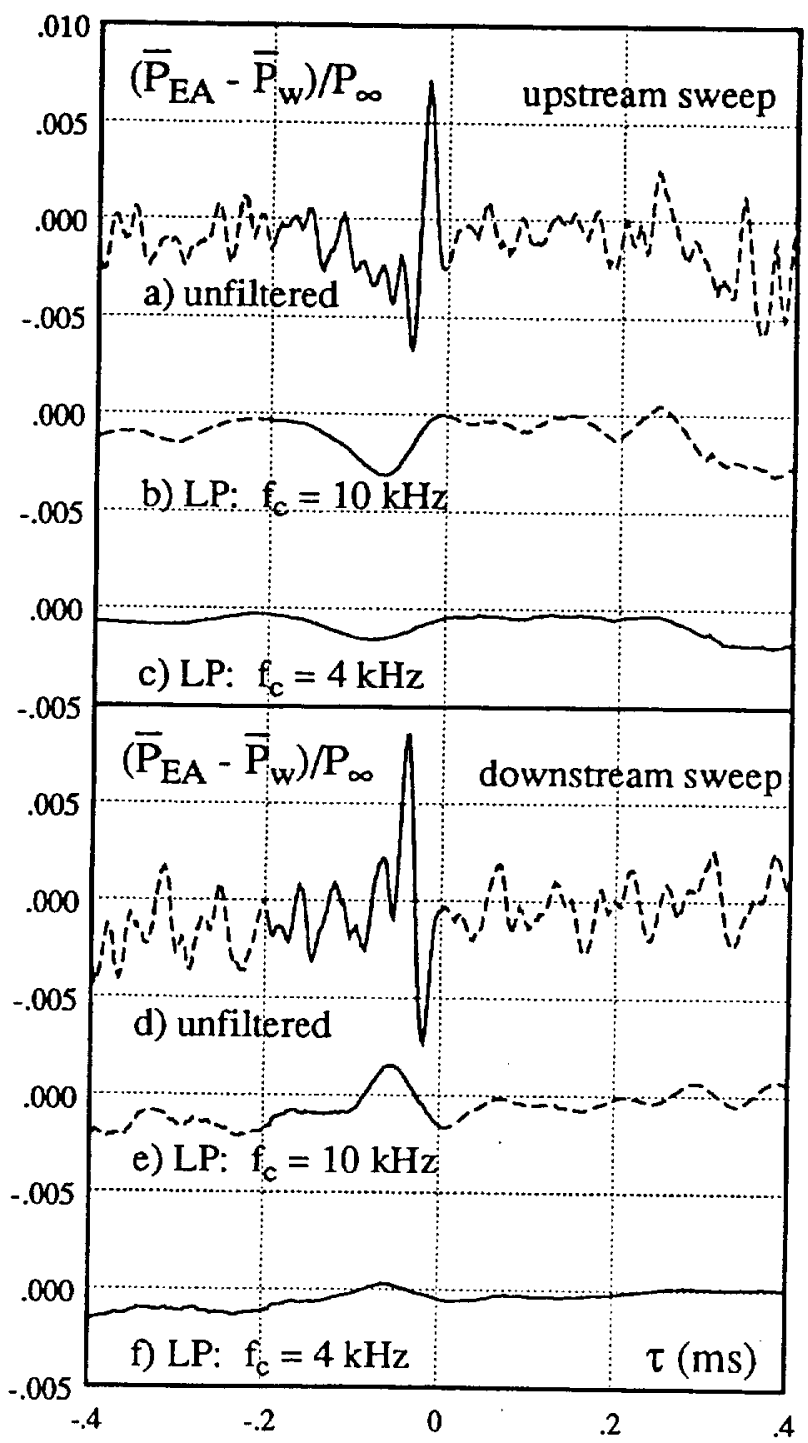

Fig 12 Digital filtering of UTBL ensemble average. 


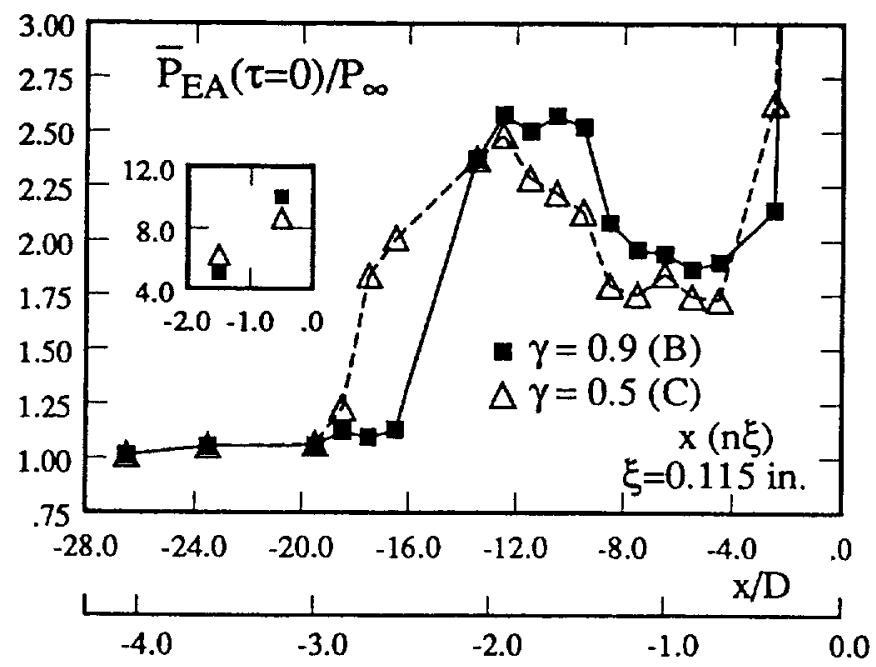

Fig 13 Shock foot fixed ensemble average pressure distributions. 


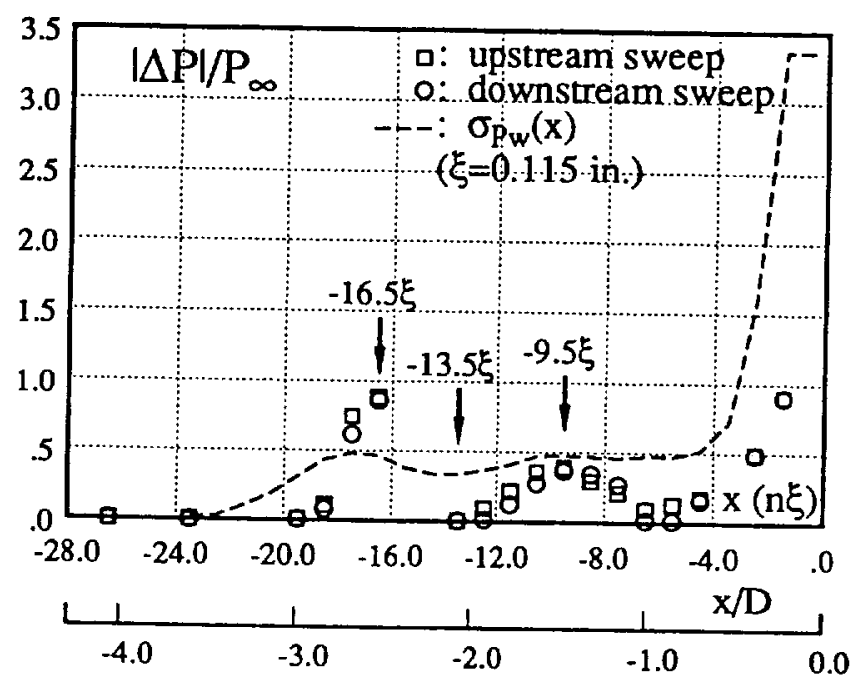

Fig 14 Predicted standard deviation distribution. 


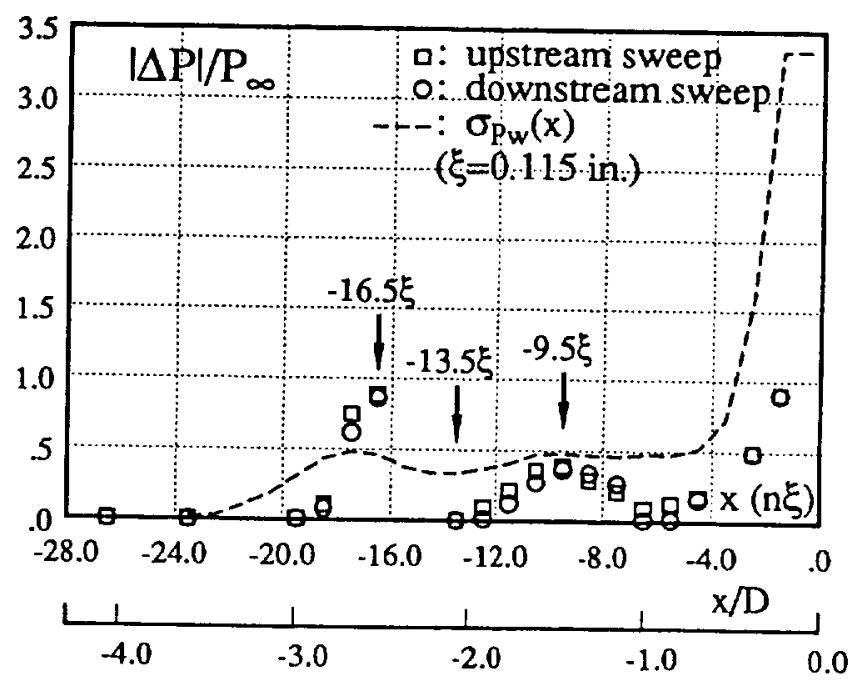

Fig 14 Predicted standard deviation distribution. 


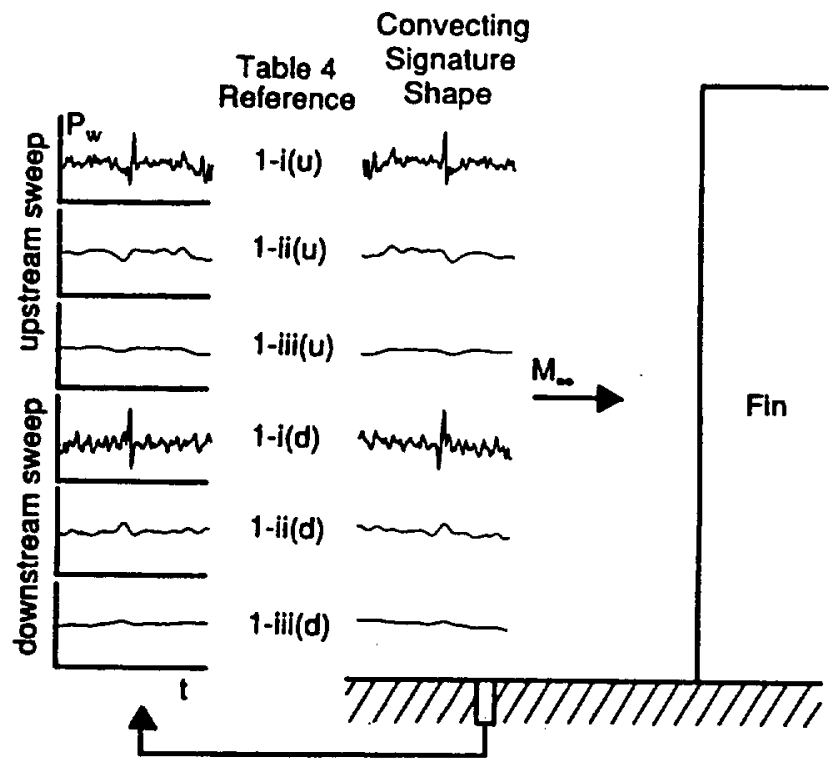

Fig 15 Physical characteristics of UTBL convecting signals. 


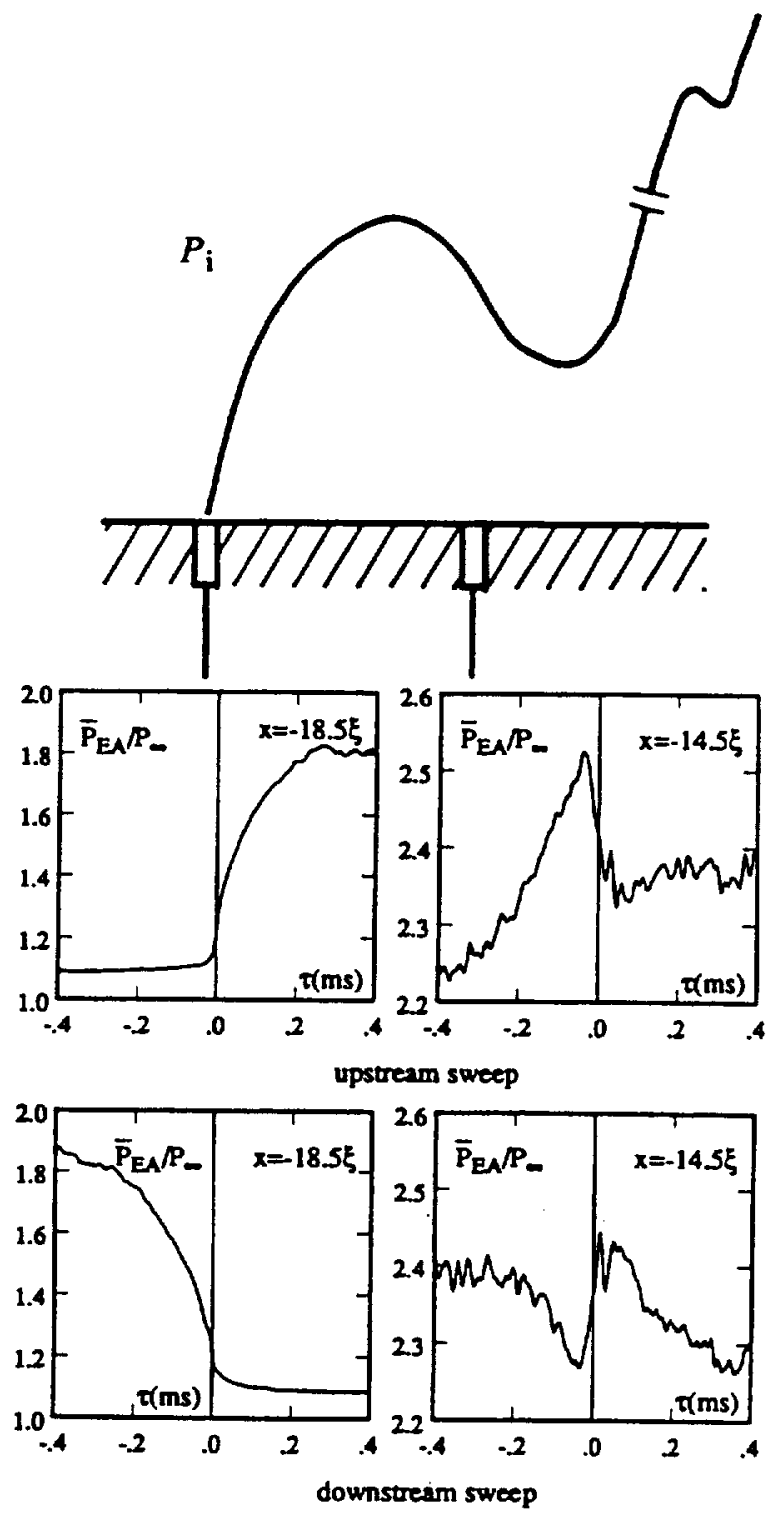

Fig 16 Ensemble average results in terms of $P_{\mathrm{j}}$. 

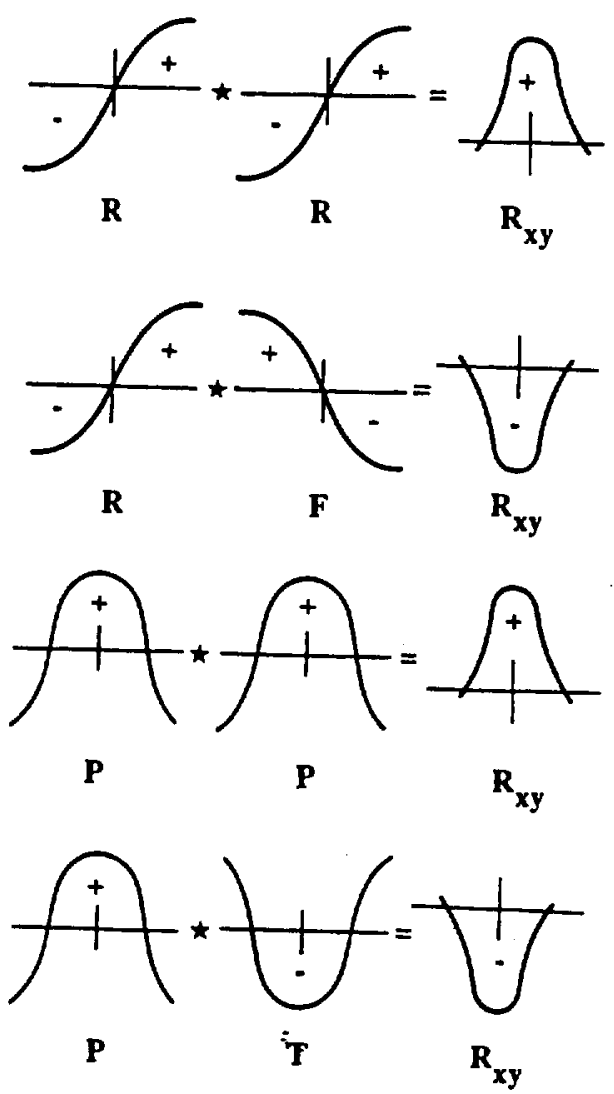

Fig 17 Qualitative model signal cross-correlation results. 


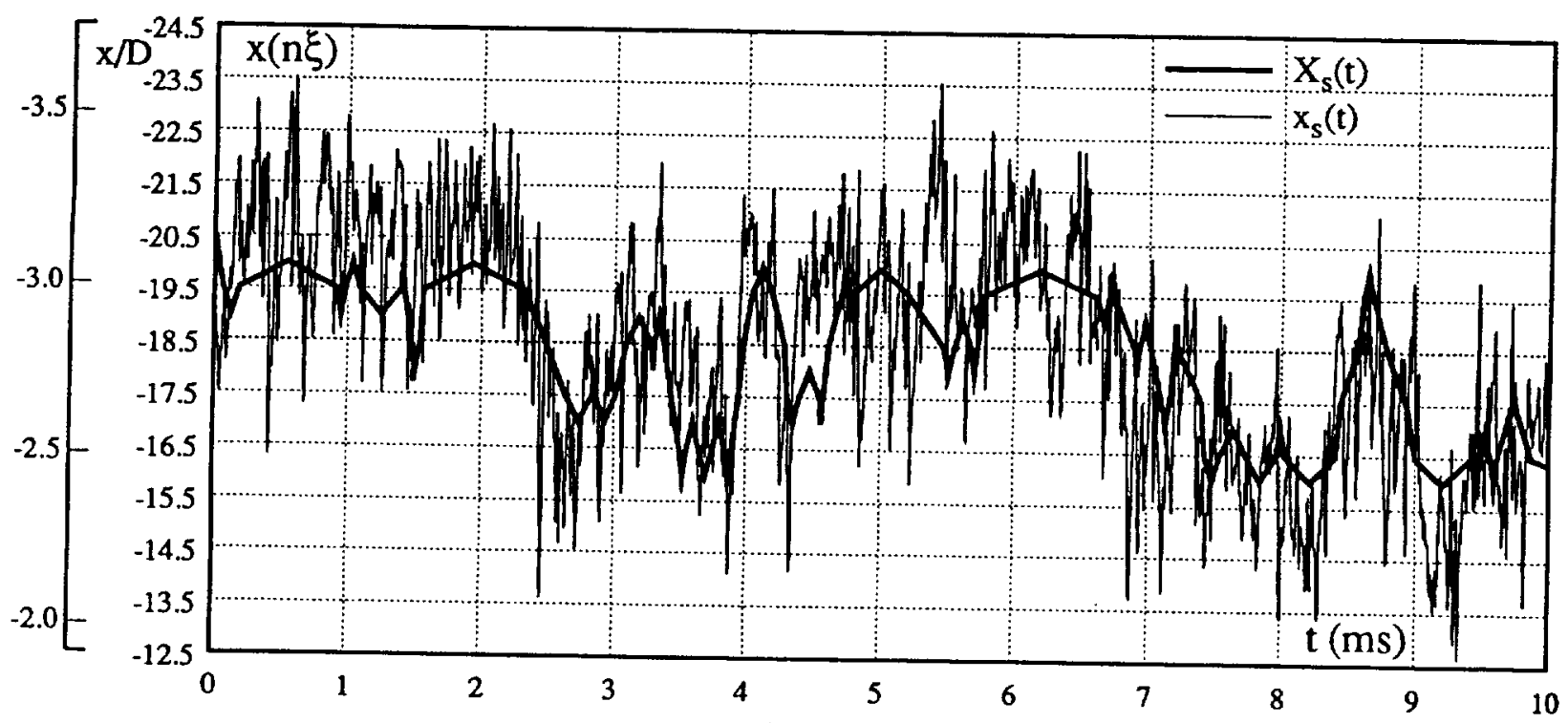

Fig 18 Actual $\left(\mathrm{X}_{\mathrm{s}}(\mathrm{t})\right)$ and predicted $\left(\mathrm{x}_{\mathrm{s}}(\mathrm{t})\right)$ shock foot position-time history (sample). 


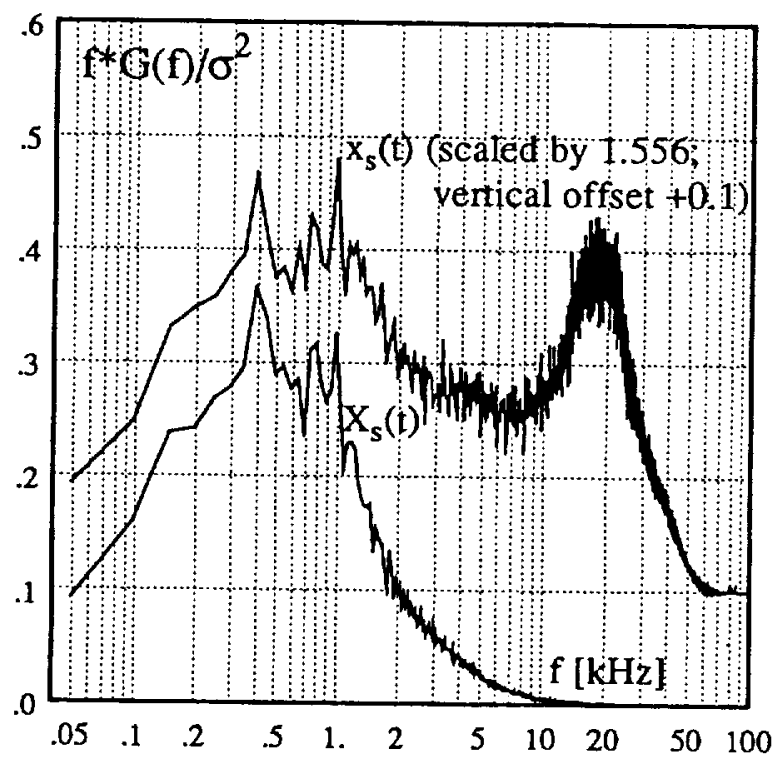

Fig $19 X_{s}(t), x_{s}(t)$ power spectra. 


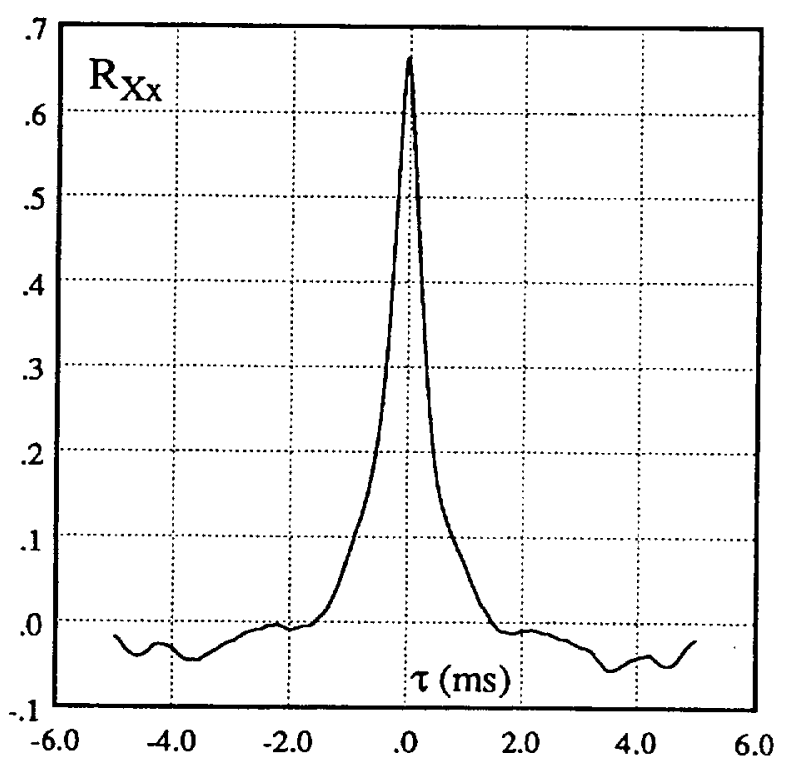

Fig $20 X_{s}(t), x_{s}(t)$ cross-correlation. 


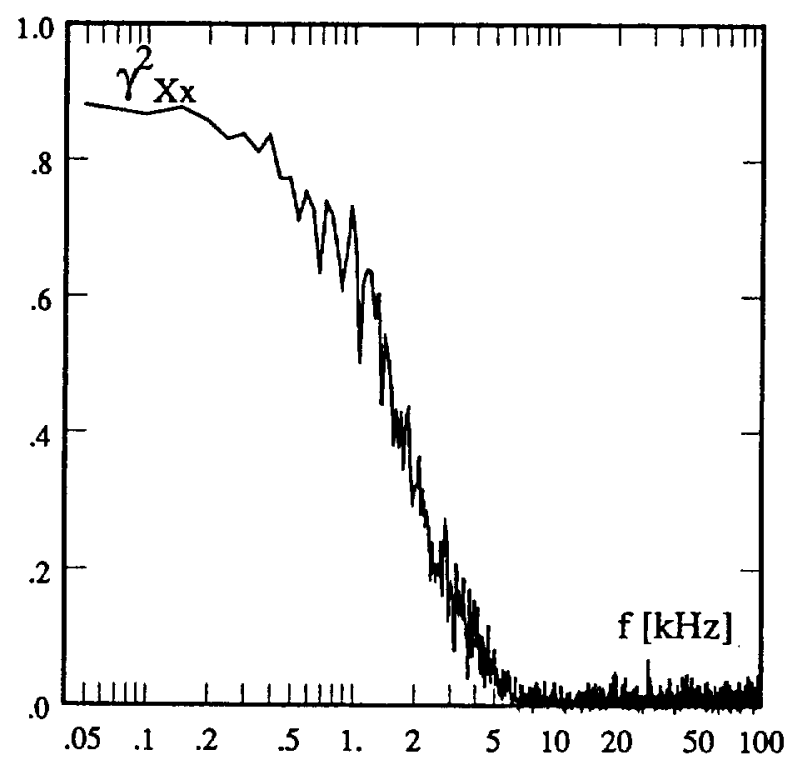

Fig $21 X_{s}(t), x_{s}(t)$ coherence function. 

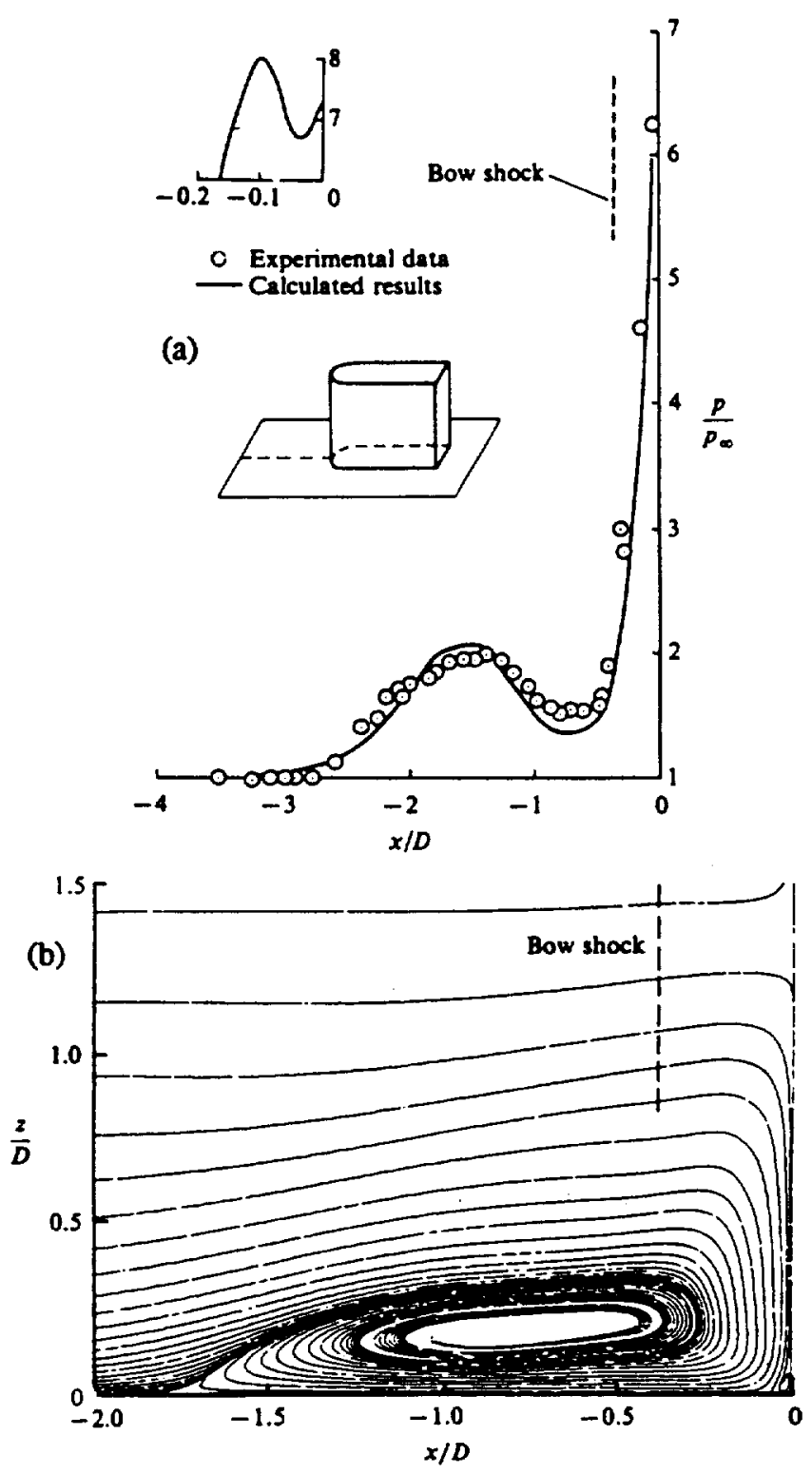

Fig 22 (a) Mach 3 computed mean pressure distribution; (b) particle paths in plane of symmetry (from Hung and Buning (1985)). 